\title{
DNA and surfactants in bulk and at interfaces
}

\author{
Rita S. Dias ${ }^{\mathrm{a}, \mathrm{b}, *}$, Alberto A.C.C. Pais ${ }^{\mathrm{a}}$, Maria G. Miguel ${ }^{\mathrm{a}}$, Björn Lindman ${ }^{\mathrm{a}, \mathrm{b}}$ \\ a Department of Chemistry, University of Coimbra, 3004-535 Coimbra, Portugal \\ ${ }^{\mathrm{b}}$ Department of Physical Chemistry 1, Centre for Chemistry and Chemical Engineering, \\ Lund University, P.O. Box 124, S-22100 Lund, Sweden
}

Received 13 July 2004; accepted 29 July 2004

\begin{abstract}
Recent investigations of the DNA interactions with cationic surfactants and catanionic mixtures are reviewed. Several techniques have been used such as fluorescence microscopy, dynamic light scattering, electron microscopy, and Monte Carlo simulations.

The conformational behaviour of large DNA molecules in the presence of cationic surfactant was followed by fluorescence microscopy and also by dynamic light scattering. These techniques were in good agreement and it was possible to observe a discrete transition from extended coils to collapsed globules and their coexistence for intermediate amphiphile concentrations. The dependence on the surfactant alkyl chain was also monitored by fluorescence microscopy and, as expected, lower concentrations of the more hydrophobic surfactant were required to induce DNA compaction, although an excess of positive charges was still required.

Monte Carlo simulations on the compaction of a medium size polyanion with shorter polycations were performed. The polyanion chain suffers a sudden collapse as a function of the concentration of condensing agent, and of the number of charges on the polycation molecules. Further increase in the concentration increases the degree of compaction. The compaction was found to be associated with the polycations promoting bridging between different sites of the polyanion. When the total charge of the polycations was lower than that of the polyanion, a significant translational motion of the compacting agent along the polyanion was observed, producing only a small-degree of intrachain segregation, which can explain the excess of positive charges necessary to compact DNA.

Dissociation of the DNA-cationic surfactant complexes and a concomitant release of DNA was achieved by addition of anionic surfactants. The unfolding of DNA molecules, previously compacted with cationic surfactant, was shown to be strongly dependent on the anionic surfactant chain length; lower amounts of a longer chain surfactant were needed to release DNA into solution. On the other hand, no dependence on the hydrophobicity of the compacting agent was observed. The structures of the aggregates formed by the two surfactants, after the interaction with DNA, were imaged by cryogenic transmission electron microscopy. It is possible to predict the structure of the aggregates formed by the surfactants, like vesicles, from the phase behaviour of the mixed surfactant systems.

Studies on the interactions between DNA and catanionic mixtures were also performed. It was observed that DNA does not interact with negatively charged vesicles, even though they carry positive amphiphiles; however, in the presence of positively charged vesicles, DNA molecules compact and adsorb on their surface.

Finally Monte Carlo simulations were performed on the adsorption of a polyelectrolyte on catanionic surfaces. It was observed that the mobile charges in the surface react to the presence of the polyelectrolyte enabling a strong degree of adsorption even though the membrane was globally neutral. Our observations indicate that the adsorption behaviour of the polyelectrolyte is influenced by the response given by the membrane to its presence and that the number of adsorbed beads increases drastically with the increase of flexibility of the polymer. Calculations involving polymers with three different intrinsic stiffnesses showed that the variation is non-monotonic. It was observed also that a smaller polyanion typically adsorbs more completely than the larger one, which indicates that the polarisation of the membrane becomes less facilitated as the degree of disruption increases.
\end{abstract}

(c) 2004 Elsevier B.V. All rights reserved.

Keywords: DNA-surfactant interactions; Monte Carlo simulations; Catanionic mixtures; DNA adsorption

* Corresponding author

E-mail address: rita.dias@fkem1.lu.se (R.S. Dias). 


\section{Introduction}

The compaction of DNA, together with the reduction of its charges, is believed to facilitate the uptake of nucleic acids through the cellular membrane [1-5]. Since the strong binding of cationic surfactants to DNA allows these two effects to be fulfilled, it is not surprising that the complexation with cationic lipids is one strategy for delivery of DNA to cells. However, synthetic cationic surfactants per se cannot be used for this purpose, since the complexes of DNA and cationic micelles do not result in effective transfection. It is a common viewpoint to explain this low transfection by the cytotoxicity of surfactants and a low stability of these complexes upon a change in the environment [6]. In spite of this, quaternary ammonium surfactants can be used, in small amounts, for charging of neutral liposomes, thereby improving their transfection efficiency; they have the advantage of lower cost when compared with other synthetic lipids [6,7]. The degree of compaction is not often discussed but it is believed to be important for the delivery of DNA to cells. Interestingly, and opposite to what could be expected, not always the most compact complexes are the most efficient [8].

After delivery, DNA must become accessible to the enzymatic machinery of the cell. Since lipid complexation is known to inhibit at least certain DNA processing enzymes such as DNAse [9-11], it is likely that the transfected DNA can become active only by release from the lipid complex. In vitro such release can be accomplished by addition of anionic species, like surfactants, which bind the cationic lipid and release DNA [12,13], and there are indications that such a mechanism may play a role also in vivo [14], at least for oligonucleotides. Bhattacharya and Mandal [12] have shown by circular dichroism, electrophoresis and the DNAse protection assay, that after release DNA is in its native B-form.

Because of the growing interest in this field and numerous applications of the DNA-cationic surfactant systems, several studies have been presented in the literature. The strong association displayed by DNA and cationic surfactant systems is well-known, and it is related to some applications: in 1967 a procedure was first described [15] that used quaternary ammonium surfactants to precipitate DNA for its extraction and purification.

Binding of cationic surfactant to DNA occurs at concentrations well below the CMC of the surfactant and the binding isotherms have a sigmoidal shape which demonstrates the cooperative binding [16-19]. The binding isotherms were shown to be strongly dependent on the surfactant chain length [16], suggesting that hydrophobic interactions are important for the interaction and that it was analogous to the formation of micelles. Also, the binding constants were shown to change with the salt concentration $[16,17]$, indicating strong electrostatic interactions between the negatively charged DNA and the oppositely charged surfactants.

A number of studies have been presented on the DNA-cationic surfactant complex formation [18,20-22], precipitation [23], and microstructure [24-29].
Studies on the dilute regime have also been performed. Fluorescence microscopy studies on large DNA molecules, consisting of several kilo base pairs (kbp), have shown that cationic surfactants induce a discrete collapse from DNA coil to a compact globular form. For intermediate concentrations of surfactant a region is observed where both DNA coils and globules coexist [19]. This coexistence region is a common phenomenon for DNA molecules on the addition of condensing agents such as organic solvents [30], flexible polymers [31], and multivalent ions [32-34]. The coil-globule transition of long DNA molecules is then discrete, a (quasi-) firstorder transition for individual chains, but continuous for their ensemble average [30,32]. Compaction of DNA is believed to be driven by attractive interactions between different parts of the molecule, by ion correlation effects arising from the presence of multivalent ions, for example [35,36], leading to the formation of a nucleation centre in the DNA chain that grows along the molecule chain [37]. Due to the hydrophobic interactions between the cationic surfactant molecules, these will self-assemble and act as multivalent ions, inducing DNA compaction.

The compaction and packing of DNA in cells is of biological importance. Histones, spherical positively charged proteins, are mainly responsible for it [38-40]. However a series of other positively charged agents within the cell are believed to also play a role. Polyamines, like spermidine and spermine, constitute a group of cell components and even though their functions are mostly unknown, it is believed that they are important in the regulation of cell proliferation and differentiation. Since they are always found in association with nucleic acids it is also widely accepted that they act as helpers in DNA packaging.

The interactions between polyamines and DNA started to be studied some time ago $[41,42]$ and the interest for these systems has grown steadily. Polyamines interact predominantly by electrostatic interactions with DNA molecules [43] inducing their compaction [44], aggregation [45] and precipitation [46,47]. Although these systems have been much studied for decades, there are a number of interesting points which only recently have been explained. Spermine, for example, is used in high concentrations as crystallizing agents; nevertheless, spermine molecules are usually not detected in X-ray studies. It has been proposed recently [48] that the flexible polyamine molecules interact in an irregular manner with the DNA molecule, with no definite binding sites.

Fluorescence microscopy has also been used to study the interactions between DNA and polyamines [49,50], and other related agents such as chitosans [52].

The phenomenon of polymer, or protein, adsorption in lipid monolayers or bilayers has great importance in biophysics since many biological processes occur at interfaces involving these components.

Also the adsorption of macromolecules such as DNA or proteins at planar or spherical substrates is an intermediate step in fabrication of drugs or gene delivery vehi- 
cles. One of the most studied systems of non-viral gene therapy are the so-called lipoplexes, complexes formed between DNA molecules and liposomes, vesicular structures formed typically by a mixture of a neutral lipid and a cationic one $[53,54]$. The formation of such complexes starts with the adsorption of DNA onto the positively charged membrane. These systems have been extensively studied and even though the mechanism of formation is still far from understood, the structure of the complexes is believed to be a short ranged lamellar, composed of amphiphile bilayers with DNA molecules ordered and packed between the lipid stacks. This type of structure has been observed for systems with different lipid components [55-58].

This, and the fact that DNA, with its unique structure, can act as a good candidate for future nanodevices like templates, biosensors, or semi-conducting molecules, lead to a great interest in the study of DNA at interfaces by techniques like ellipsometry, atomic force microscopy, neutron reflectivity, Brewster angle microscopy, etc.

Several studies have been conducted regarding the interactions between DNA and neutral and/or cationic bilayers. Ellipsometry studies have shown that a thick layer of a few DNA molecules adsorbed on an hydrophobic surface undergoes a strong condensation into a thin and denser layer by the addition of cationic surfactant $[59,60]$. DNA adsorption on cationic lipid bilayers was also studied by atomic force microscopy and the large DNA molecules were shown to destabilize the membrane [61]. Also the adsorption was followed by surface plasmon spectroscopy [62] and by means of fluorescence microscopy and it was found that the molecules, when confined in two-dimensions adsorb in an extended conformation [63,64].

Due to the obvious interest in this field the adsorption of a polymer onto substrates have also been subject of many simulation studies (for review see Ref. [65]). Studies on the adsorption of a charged polymer onto an oppositely charged homogeneous surface are the most abundant and the phenomena involved reasonably well understood.

In a lamellar phase, when in its fluid state, the lipids present a relatively fast lateral diffusion, which are responsive to the approach of charged objects.

In fact this demixing of the lipids and formation of domains in mixed lipid membranes has been observed recently in fluorescence microscopy experiments on the adsorption of DNA [63] and proteins [66] on membranes.

Recently some molecular simulation studies on heterogeneous surfaces, with patches of different charges and charge densities [67,68], and theoretical studies involving mobile ions [69] show a clear attempt of mimicking real systems. However, and specially in the molecular simulations, studies on the interaction between a polyelectrolyte and a membrane with "real" characteristics (possibility for movement of the particles within the membrane, inherent flexibility) are lacking in the literature.

\section{Compaction of DNA with cationic surfactant}

Despite the interest on interaction of DNA with cationic surfactants and other compacting agents, there is a lack of studies in the literature about the dilute regime, maybe due to the difficulty in finding suitable techniques. We believe these studies to be important since they give complementary insight to the interaction mechanisms by allowing the studies on the intramolecular level. Here we present a study in this regime, based on fluorescence microscopy, dynamic light scattering and Monte Carlo simulations.

\subsection{A fluorescence microscopy study}

Fluorescence microscopy (FM) is a technique that has recently started to be used in the study of DNA conformational behaviour in the presence of various cosolutes, and its main advantage is to allow for the visualization of single molecules in solution.

DNA molecules in aqueous solution present an extended conformation, migrating in the solution and exhibiting a relatively slow worm-like motion, i.e., they are in the unfolded coil conformation. When a cationic surfactant like, for example, tetradecyltrimethylammonium bromide (TTAB) is added to the DNA solution above a certain concentration, in this case $2.0 \times 10^{-5} \mathrm{M}$, we observe a coexistence region of some compact molecules in solution along with DNA coils. These compact molecules, that present a high fluorescence intensity, and a long-axis length less than $1.0 \mu \mathrm{m}$, are denoted as DNA globules. With further increase of the surfactant concentration, $[\mathrm{TTAB}]=8.0 \times 10^{-5} \mathrm{M}$, we reach a region where only DNA globules are detected. In Fig. 1 we represent this conformational change by plotting the long axis length of the DNA molecules versus the surfactant concentration.

These results along with those for DNA conformational behaviour in the presence of cetyltrimethylammonium bromide (CTAB) and dodecyltrimethylammonium bromide

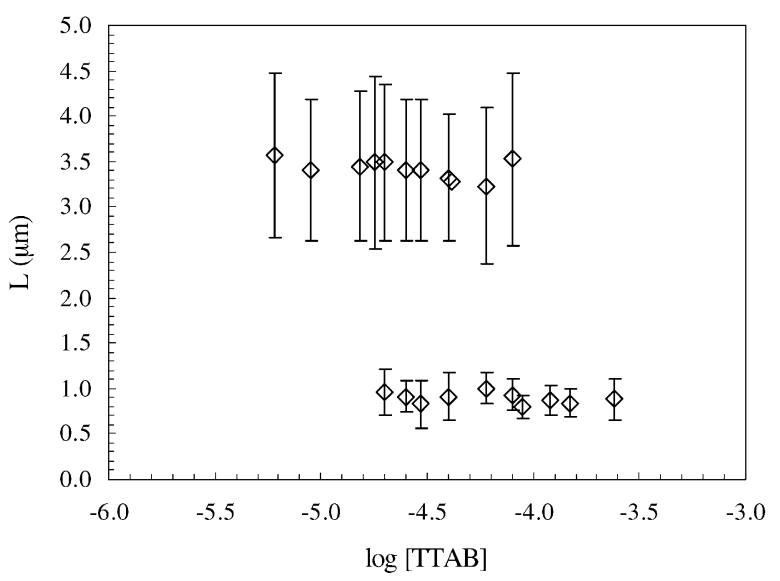

Fig. 1. Long-axis length, $L$, of T4DNA molecules, $0.5 \mu \mathrm{M}$, vs. the concentration of TTAB. Error bars indicate the statistical error in the distribution and are given by the standard deviation. $T=25^{\circ} \mathrm{C}$. From Ref. [96]. 
Table 1

Characterization of the interaction between DNA and cationic surfactants

\begin{tabular}{lccc}
\hline & $C_{0}(\mu \mathrm{M})$ & $C_{1}(\mu \mathrm{M})$ & $\Delta C(\mu \mathrm{M})$ \\
\hline CTAB & 8.0 & 24.0 & 16.0 \\
TTAB & 20.0 & 80.0 & 60.0 \\
DTAB & 80.0 & 300.0 & 220.0 \\
\hline
\end{tabular}

$C_{0}$ represents the concentration at which globules were first detected in the solution, and $C_{1}$ the disappearance of a last DNA coil. $\Delta C$ is the coexistence interval width. $T=25^{\circ} \mathrm{C}$. From Ref. [23].

(DTAB), with 16 and 12 carbons in the alkyl chain, are presented in Table 1. By looking at the conformational behaviour of DNA molecules in the presence of cationic surfactants of different chain lengths (Fig. 2), we firstly conclude that a larger amount of the shorter chain-length surfactant is needed to induce compaction of DNA macromolecules. We observed that the coexistence region begins for concentrations of 8.0 and $80.0 \mu \mathrm{M}$ for CTAB and DTAB, respectively. These results are in line with our previous results of phase map studies, where we observed that lower concentrations of the more hydrophobic surfactant are needed to induce the precipitation of the system [23].

Compaction is driven by attractive electrostatic interactions between different parts of a DNA double helix due to the correlation effects arising in the presence of multivalent counter-ions $[35,30]$. Surfactants have only one charge per molecule but, due to their self-assembly properties, form micellar aggregates in the vicinity of the oppositely charged macromolecule at a certain critical concentration [70], that act as multivalent ions. This is evident from the fact that $\mathrm{CTAB}$, the longer-chained surfactant, is more efficient compacting DNA than the shorter-chained surfactant.

One interesting observation allowed by the used technique is the critical collapse of the DNA molecules, without the apparent existence of intermediate structures, and the coexistence of extended and collapsed molecules. This coexistence, common to most compacting agents, has only been observed, to our knowledge by FM and atomic force microscopy (AFM), and one should keep in mind that the complexation at interfaces differs from the complexation in the bulk. We

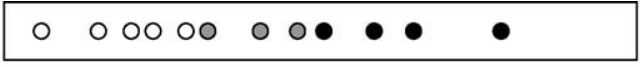

CTAB

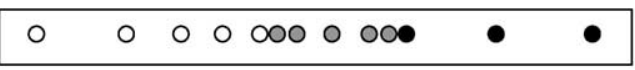

TTAB

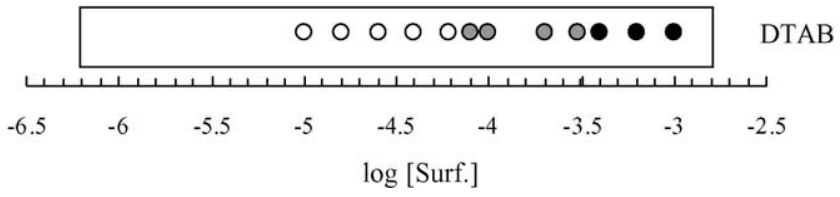

Fig. 2. DNA conformational behaviour in the presence of cationic surfactants CTAB, TTAB, and DTAB. The DNA charge concentration was maintained at $0.5 \mu \mathrm{M}$. Open circles correspond to the coil conformational state of DNA and filled ones to the presence of globular DNA molecules. Shaded circles represent the coexistence between elongated coils and compacted globules. $T=25^{\circ} \mathrm{C}$. From Ref. [96].

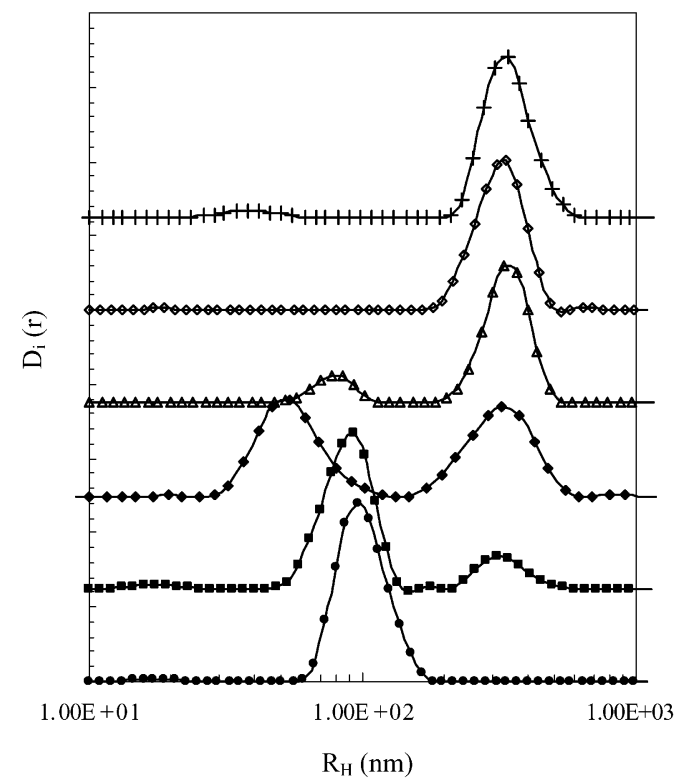

Fig. 3. Intensity weighted distribution function of $0.5 \mu \mathrm{M}$ T2DNA solution $(10 \mathrm{mM} \mathrm{NaBr})$ in the absence (upper curve) and presence of CTAB. The concentrations of the cationic surfactant are from the top curve to the bottom: 0 (only DNA), 1.0, 2.0, 6.0, 10.0, and 30.0 $\mu \mathrm{M} . \theta=90^{\circ}$ and $T=27^{\circ} \mathrm{C}$. From Ref. [97].

performed then dynamic light scattering (DLS) studies on the DNA-CTAB system on the same dilution conditions.

\subsection{A dynamic light scattering study}

Light scattering techniques are very relevant in the study of colloidal particles, specially in what concerns the size of macromolecules and molecular assemblies. While there has been a number of DLS reports on the properties of DNA solutions (see, for example Refs. [71-74]), studies involving interactions between DNA and cationic amphiphiles are in a very limited number $[18,24,75]$.

We started by performing DLS measurements on the DNA and surfactant (CTAB) solutions independently. For the DNA solutions the average scattered intensity was very low. In spite of the fact that the DNA used in these experiments is very large (T2DNA with $164 \mathrm{kbp}$ ) its concentration was very low, $0.5 \mu \mathrm{M}$ in phosphate groups; thus we had no interactions between the molecules. The intensity weighted correlation function of the DNA solution (upper curve in Fig. 3) presented only one peak, corresponding to the translational mode of the molecules giving a hydrodynamic radius of about $330 \mathrm{~nm}$. For CTAB solutions the scattering intensity was also very low and we found no significant peaks for solutions with the maximum concentration used in this work.

When the cationic surfactant is added to the DNA solution in a stepwise manner, we first observe no changes and then, for a CTAB concentration of $2 \mu \mathrm{M}$, we observe the appearance of a second peak corresponding to entities with lower hydrodynamic radius, of about $80 \mathrm{~nm}$. With further addition of CTAB this peak increases in amplitude while the 


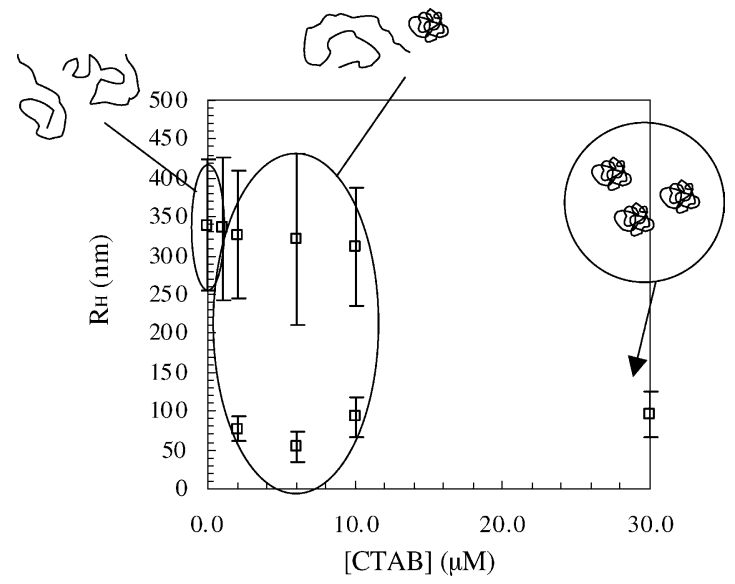

Fig. 4. Hydrodynamic radius of the DNA molecules, taken from the position of the peaks of the calculated intensity distribution functions (Fig. 3), vs. the concentration of surfactant, CTAB. The error bars represent the width of the peak at half height. The conformation of the DNA molecules is schematically depicted. From Ref. [97].

pure DNA translational mode decreases, until it disappears for surfactant concentrations of $30 \mu \mathrm{M}$ (Fig. 3).

This is a significant result since it suggests, as observed previously in FM, not a gradual change of the DNA size but the existence of two populations in the sample, one of extended DNA coils coexisting with DNA compacted molecules.

In Fig. 4 the hydrodynamic radius of the DNA molecules is represented as a function of the surfactant concentration, with data taken from the size distribution calculations (Fig. 3). If we compare this graphic with Fig. 1 we see that they are very similar. As observed for FM the coexistence of two populations of DNA molecules is evident: one due to extended molecules which present some fluctuation in their shape, i.e. size (Gaussian chain), as given by the width of the respective peak; and another consisting of compacted molecules presenting a smaller hydrodynamic radius and less fluctuations in size. The measurements performed by DLS give also an improvement relative to the FM data in what concerns the size of the DNA-surfactant aggregates, since direct FM measurements overestimate the size of the complexes, due to the blurring effect of the DNA-DAPI complex [76].

The interactions between DNA and cationic surfactants were studied by fluorescence microscopy and dynamic light scattering. We saw that DNA exhibits a discrete phase transition in the presence of cationic surfactants from coils to globules. Fluorescence microscopy experiments were made with cationic surfactants of different chain lengths and we concluded that CTAB, the longer-chained surfactant, is more efficient in compacting DNA than the shorter-chained surfactant.

Looking again at Table 1, we realize that, even though the amounts required to compact DNA were very small it should be noticed that they correspond to a large excess of positive charges when compared to the concentration of DNA nucleotides, $0.5 \mu \mathrm{M}$ in phosphate groups. This is a feature
Table 2

Characterization of the interaction between DNA $(0.1 \mu \mathrm{M}$ in phosphates $)$ and polycations of different chain lengths: 1,3-diaminopropane ( $\left.\mathrm{DA}_{3}\right)$, spermidine (SPD) and spermine (SPM)

\begin{tabular}{lcc}
\hline & $C_{0}(\mu \mathrm{M})$ & $C_{1}(\mu \mathrm{M})$ \\
\hline $\mathrm{DA}_{3}\left(\mathrm{M}^{2+}\right)$ & 400 & 1600 \\
$\mathrm{SPD}\left(\mathrm{M}^{3+}\right)$ & 80 & 120 \\
$\mathrm{SPM}\left(\mathrm{M}^{4+}\right)$ & 8 & 12 \\
$\mathrm{P}$-arginine $(\mathrm{DP}=120)$ & - & $0.5^{\mathrm{a}}$ \\
\hline$C_{0}$ represents the concentration at which globules were first detected in the \\
solution, and $C_{1}$ the disappearance of a last DNA coil. $T=25^{\circ} \mathrm{C}$. Drawn \\
from Ref. [50]. \\
$\quad$ a The presented value does not correspond to concentration of compacting \\
agent but to the mixing ratio of positive and negative charges, $\beta$. From Ref. \\
[49].
\end{tabular}

common to other systems. Table 2 displays some results of fluorescence microscopy studies presented in the literature. It was observed that in the presence of polyamines DNA undergoes a discrete conformational change from coil to globule and that, even with spermine, having four charges per molecule, an excess of positive charges was required for the compaction to initiate. Furthermore, the concentrations of the polycations required to induce compaction of DNA decrease substantially with the increase of the charges in the chain, differing by about one order of magnitude [50,51]. When longer polycations were used, charge neutralization was not even required [49].

\section{Modelling of DNA compaction by polycations}

It was already mentioned the importance of the understanding of DNA compaction in cells. Even though histones, are the responsible for the packing in most of the cells, polyamines like spermidine and spermine appear associated with DNA and are believed to play a role in its compaction. It is curious that the most compact form of DNA is found in sperm heads where the condensing agents are positively charged polymers.

The degree of compaction is an interesting property, known to be of major importance for gene therapy and transfection efficiencies [5,8]. Varying degrees of compaction have been predicted through molecular dynamics simulations for oppositely charged chains of the same length [77], depending on the interaction strength. Also, the characterization of complexes in solutions containing positive and negative chains has been achieved on the basis of Monte Carlo simulations $[78,79]$, showing that the nature of the complexes varies with the linear charge density of the chains.

Here we report on a study of polycations as compacting agents of a medium size polyanion by means of Monte Carlo simulations. The systems under study are summarised in Table 3.

The degree of compaction was determined on varying the size of the positive chains and their number. The systems are characterized using a conformational analysis in which 
Table 3

Systems studied in the present work

\begin{tabular}{lccc}
\hline System & \multicolumn{2}{l}{ Number of } & \\
\cline { 2 - 4 } & Positive chains & Beads/chains & Counter-ions \\
\hline$(0 p+0)$ & 0 & 0 & 120 \\
$(40 p+3)$ & 40 & 3 & 0 \\
$(30 p+4)$ & 30 & 4 & 0 \\
$(24 p+5)$ & 24 & 5 & 0 \\
$(12 p+10)$ & 12 & 10 & 0 \\
$(8 p+15)$ & 8 & 15 & 0 \\
$(1 p+30)$ & 1 & 30 & 90 \\
$(2 p+30)$ & 2 & 30 & 60 \\
$(3 p+30)$ & 3 & 30 & 0 \\
$(4 p+30)$ & 4 & 30 & 0 \\
\hline
\end{tabular}

In all cases, a negative polyelectrolyte with 12 beads of unit charge is present. Counter-ions are positive. The nomenclature of the systems is the following (no. of polycation chains) $\mathrm{p}+$ (no. of beads in each chain).

shape, overall dimensions, and contact of the polyanion with the compaction agents is taken into consideration.

\subsection{Effect of the chain length}

We started by looking at the effect of the positive polymer chain lengths on the conformation of the long negative chain.

The average properties of the negative polyelectrolyte chains are shown in Table 4, for all systems considered in this work.

We can see that, for the larger values of the polycation chain length, a significant degree of compaction is attained. The end-to-end distance $\left\langle R_{\text {ee }}^{2}\right\rangle^{1 / 2}$, radius of gyration $\left\langle R_{\mathrm{g}}^{2}\right\rangle^{1 / 2}$ and persistence length, $l_{\mathrm{p}}$, significantly decrease when the number of positive beads is increased in each positive chain. The latter is calculated by using the results obtained in the simulation for the radius of gyration through the relationship [80],

$\left\langle R_{\mathrm{g}}^{2}\right\rangle=\frac{L l_{\mathrm{p}}}{3}-l_{\mathrm{p}}^{2}+2 \frac{l_{\mathrm{p}}^{3}}{L}-2 \frac{l_{\mathrm{p}}^{4}}{L^{2}}\left[1-\exp \left(\frac{-L}{l_{\mathrm{p}}}\right)\right]$

where $L$ corresponds to the contour length.

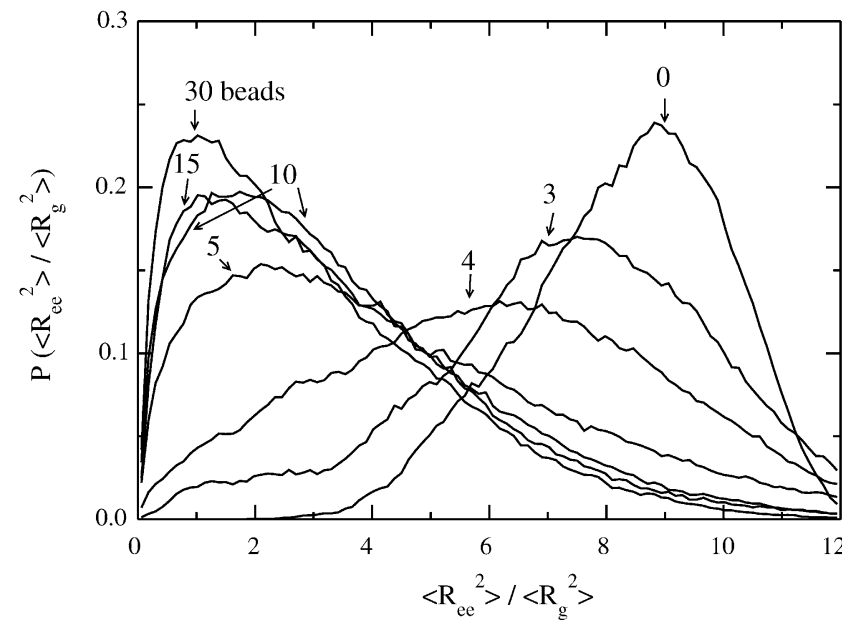

Fig. 5. Shape $\left\langle R_{\mathrm{ee}}^{2}\right\rangle /\left\langle R_{\mathrm{g}}^{2}\right\rangle$ distribution functions for systems $(0 \mathrm{p}+0)$, $(40 \mathrm{p}+3),(30 \mathrm{p}+4),(24 \mathrm{p}+5),(12 \mathrm{p}+10),(8 \mathrm{p}+15)$ and $(4 \mathrm{p}+30)$. Curves are labelled according to the number of beads in the polycation; when the polyanion is in the presence of monovalent counter-ions only, we use the label 0. From Ref. [98].

For the shorter chains, the polyelectrolyte behaves close to a Gaussian coil, as shown by the average value of the shape factor, $\left\langle R_{\mathrm{ee}}^{2}\right\rangle /\left\langle R_{\mathrm{g}}^{2}\right\rangle$. As the polycation length increases, this factor decreases to values compatible with compact spherelike structures.

We recall that this factor has the value 12 for a rigid rod, 6 for a Gaussian chain and values below 3 for compact structures. Judging from the results presented in Table 3, no compaction occurs for the number of beads being equal to or less than 4 and $\beta=$ [no. polycation beads] $/[$ no. polyanion beads $]=1$. However, we can see that above this number of polycation beads, the polyelectrolyte chain tends to fold without a significant change in the average sequential bead angle, $\langle\alpha\rangle$. This leads to the conclusion that compact structures are obtained not through direct compression of the chain, via bending modes, but through torsions and long-range correlations. This is analogous to the observation [80,81] that chains stiffened by electrostatic interactions retain a higher

Table 4

Average properties of the negative polyelectrolyte in the different systems (Table 3)

\begin{tabular}{|c|c|c|c|c|c|}
\hline System & $\langle\alpha\rangle$ & $\left\langle R_{\mathrm{ee}}^{2}\right\rangle^{1 / 2}$ & $\left\langle R_{\mathrm{g}}^{2}\right\rangle^{1 / 2}$ & $\left\langle R_{\mathrm{ee}}^{2}\right\rangle /\left\langle R_{\mathrm{g}}^{2}\right\rangle$ & $l_{\mathrm{p}}$ \\
\hline$(0 p+0)$ & $139.1 \pm 0.1$ & $379 \pm 6.8$ & $130 \pm 1.4$ & $8.4 \pm 0.16$ & $106 \pm 3.6$ \\
\hline$(40 p+3)$ & $129.7 \pm 0.1$ & $177 \pm 7.1$ & $64 \pm 2.3$ & $7.4 \pm 0.39$ & $19 \pm 1.5$ \\
\hline$(30 p+4)$ & $128.4 \pm 0.1$ & $116 \pm 5.6$ & $46 \pm 1.3$ & $6.3 \pm 0.39$ & $10 \pm 0.6$ \\
\hline$(24 p+5)$ & $127.9 \pm 0.1$ & $68 \pm 4.3$ & $31 \pm 0.7$ & $4.5 \pm 0.42$ & $4.4 \pm 0.2$ \\
\hline$(12 p+10)$ & $127.3 \pm 0.1$ & $50 \pm 1.4$ & $27 \pm 0.5$ & $3.5 \pm 0.20$ & $3.3 \pm 0.1$ \\
\hline$(8 p+15)$ & $127.0 \pm 0.1$ & $51 \pm 1.9$ & $27 \pm 0.6$ & $3.6 \pm 0.24$ & $3.2 \pm 0.1$ \\
\hline$(1 p+30)$ & $134.9 \pm 0.1$ & $358 \pm 3.5$ & $112 \pm 1.2$ & $10.2 \pm 0.24$ & $70 \pm 0.2$ \\
\hline$(2 p+30)$ & $130.6 \pm 0.1$ & $279 \pm 3.9$ & $90 \pm 0.9$ & $9.6 \pm 0.16$ & $42 \pm 1.0$ \\
\hline$(3 p+30)$ & $128.3 \pm 0.1$ & $202 \pm 6.8$ & $73 \pm 2.1$ & $7.5 \pm 0.19$ & $26 \pm 1.8$ \\
\hline$(4 p+30)$ & $127.0 \pm 0.1$ & $43 \pm 1.1$ & $25 \pm 2.1$ & $3.1 \pm 0.15$ & $2.7 \pm 0.0$ \\
\hline
\end{tabular}

$\langle\alpha\rangle$ is the average sequential bead angle and is given in degrees. $\left\langle R_{\mathrm{ee}}^{2}\right\rangle^{1 / 2}$ is the polymer end-to-end distance, and $\left\langle R_{\mathrm{g}}^{2}\right\rangle^{1 / 2}$ the radius of gyration. $\left\langle R_{\mathrm{ee}}^{2}\right\rangle /\left\langle R_{\mathrm{g}}^{2}\right\rangle$ is the shape factor, ratio between the end-to-end distance and the radius of gyration. Finally $l_{\mathrm{p}}$ represents the persistence length. All distances are given in $\AA$. From Ref. [98]. 


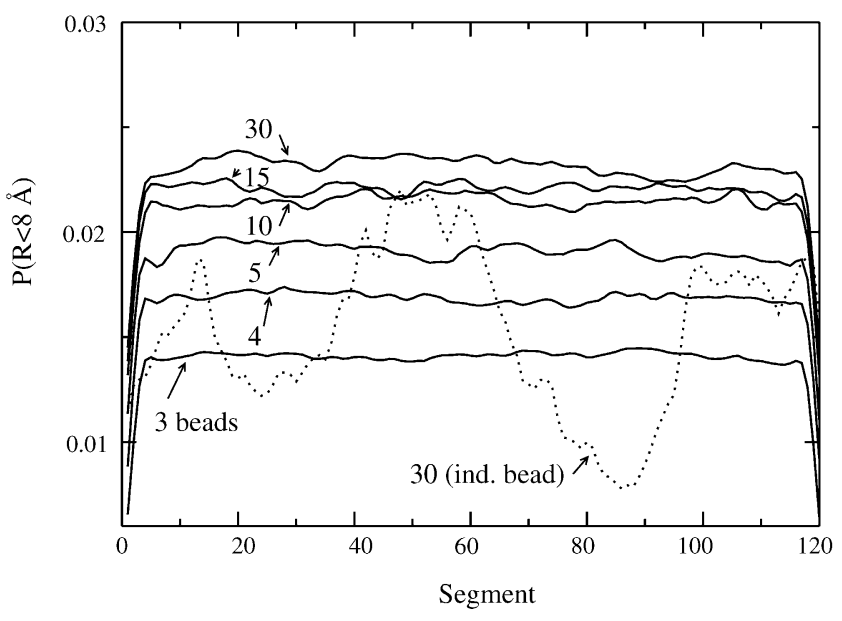

Fig. 6. Average probability of finding a polycation bead at a distance below $8 \AA$ from each negative polyelectrolyte segment. The dotted line corresponds to one randomly chosen individual positive bead. From Ref. [98]. flexibility locally, i.e., the behaviour on short length scales does not reflect the behaviour on a larger scale.

Shape factors for each of these systems are shown in Fig. 5. The probability of very compact structures drastically and suddenly increases when the polycation changes from 4 to 5 beads, although large values of this factor, significantly above 6 , are present for every system. For the longer polycations most structures are found for values well below 3 , and the largest compaction is attained for 30 beads.

Fig. 6 represents the probability of finding one positive bead, averaged over all beads, at a distance inferior of $8 \AA$ from each numbered polyanion segment, subsequently denoted simply as contact. We note that the smaller chains tend to cover uniformly the region in the proximity of the polyanion, except at the ends the probability decreases, as to be expected from a less intense electrostatic field, irrespective of the system. However, it is inferred that positive and negative chains become closer and closer as the polycation length increases.

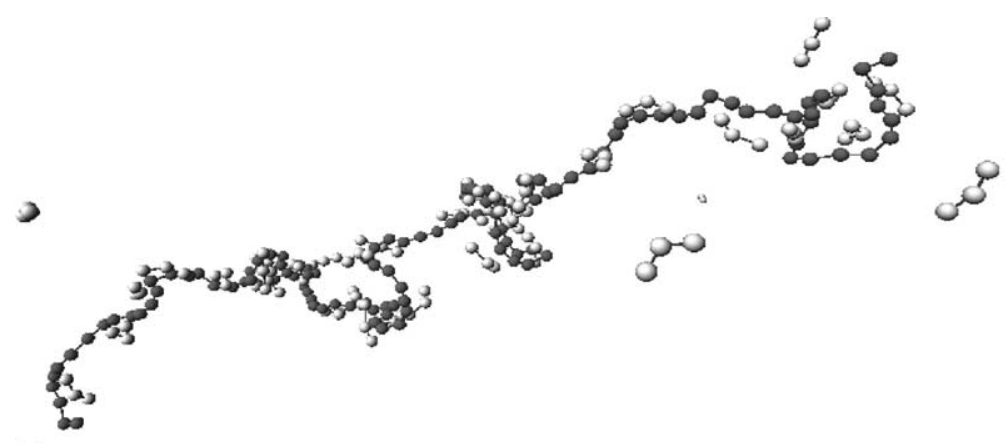

(a)

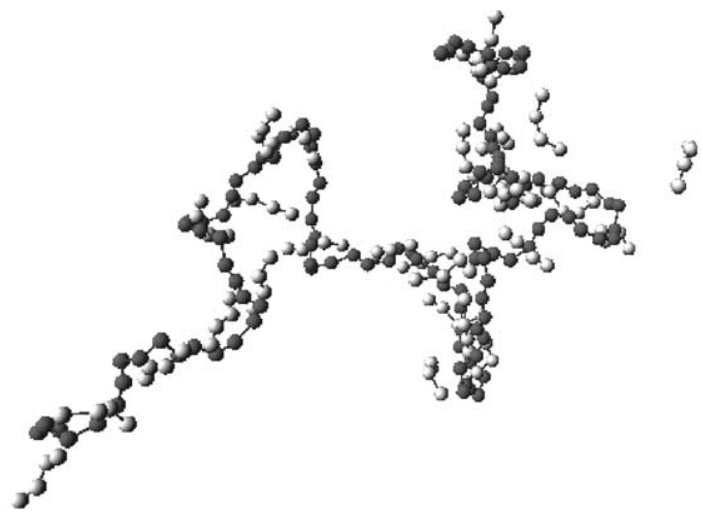

(b)
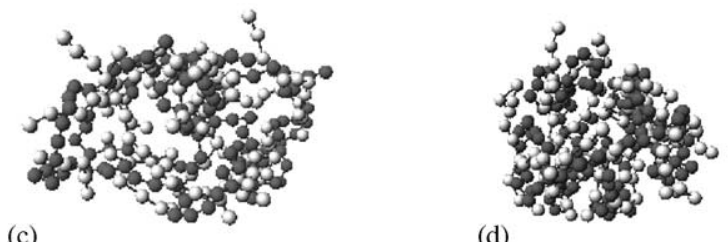

(e)

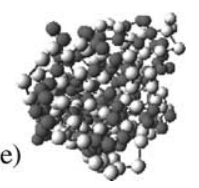

Fig. 7. Typical configurations for systems: (a) $(40 p+3)$, (b) $(30 p+4)$, (c) $(24 p+5)$, (d) $(12 p+10)$, and (e) $(4 p+30)$. From Ref. [98]. 
This effect is striking when looking at Fig. 7 which displays typical conformations for various polycation lengths. In panel (a) the polyanion displays an extended structure, with loosely bound short polycations. As we can see many positive chains are not under the direct influence of the polyanion electrostatic field and remain scattered throughout the simulation cell.

When one bead is added to the positive chains, panel (b), we can see that the polycations are somewhat closer, even though a loose binding is still noticeable, compatible with a certain mobility of the smaller chains.

Panels (b) and (c) illustrate the degree of compaction induced when the number of charges in the positive chain increases from 4 to 5. A further increase in this number of charges promotes, although to a lower extent, additional compaction in the longer chain (panels (d) and (e)). For these three last representations, all the polycations are associated with the larger polyelectrolyte. The difference between the polyanion conformation when in the presence of polycations of 4 or 5 beads is dramatic, and this sudden collapse of the longer negatively charged polymer is in line with the coil-globule transition of large DNA molecules, observed by fluorescence microscopy and dynamic light scattering.

An analysis of the contacts of each individual polycation bead with the polyanion has also been performed. A representative example is shown in Fig. 6 (dotted line), in which the probability of contact of one individual randomly chosen positive bead of a 30-bead polycation is given. It is seen that each bead interacts with a significant portion of the longer chain (typically with more than $60 \%$ of the latter) which points to an unhindered motion of the smaller chains, indicating a good coverage of the configurational space, and a fair mobility along the polyelectrolyte chain. It also shows maxima of probability for beads significantly far apart, that may be related to the fact that polycations tend to position themselves so as to promote bridging between different parts of the polyelectrolyte.

We note that interactions between DNA and spermidine or spermine have been studied by techniques like Xray diffraction [48,82], neutron scattering [83], and Raman spectroscopy [84], and by more or less complicated models $[48,51,85,86]$ but the binding properties of the polyamines to DNA are still not clear. Several types of binding have been proposed in the literature, like the location of spermine molecules in the major groove with a single contact with the polyelectrolyte [82], or that the spermine is present in higher concentration close to the minor [86]; also, that polyamines bind to DNA simply by non-specific electrostatic interactions $[83,84]$. It is, however, widely accepted that the flexible polyamines have different binding sites, interacting in an irregular way with different sites on DNA [48], forming a short-lived and non-structured [86], mobile [84] atmosphere of ions around the macromolecule. This is the proposed reason for the fact that spermine molecules, for example, are not easily detected by X-ray methods, even though they are present at a high concentration [48].
Our results, using a rather simplified model, give some interesting insight into the interactions between DNA and flexible cationic polymers. It should be noticed from Fig. 6 that compaction of the polyanion occurs when the polycations are closer to the negative chain and thus thermodynamically restrained in their motion. This can be the explanation for the fact that large amounts of spermidine and spermine are necessary to induce the compaction of DNA in single molecule experiments $[50,51]$.

\subsection{Varying the polycation/polyanion charge ratio}

In Table 4 we also present the polyanion chain properties as the number of positive chains is increased up to $\beta=1$ (systems $(1-4 p+30)$ ). We see that the clearly stretched system $(1 p+30)$ slightly folds when a new polycation is added and the same happens with a third one. However, with four polycations the chain abruptly adopts a very compact structure. Table 3 and Fig. 8 show that the average shape factor tends to increase from system $(0 p+0)$, in which the polyanion is in the presence of its small counter-ions only, to system $(l p+30)$, then slightly decreasing to system $(2 p+30)$, although above that of $(0 p+0)$. It again slightly decreases for $(3 p+30)$ and then, suddenly, presents a very small value in system $(4 p+30)$, confirming the collapse of the chain.

In Fig. 9 are presented the polyanion-polycation contact probabilities. With one polycation chain, we observe a preferential positioning in the central part of the longer chain, although a significant coverage of the polymer can also be inferred.

As before, the probability tends to decrease as we approach either end. For two positive chains, their positions are correlated and they are essentially placed at equal distances from the centre of the polyanion, which to some extent is avoided. This may be explained in terms of repulsion effects.

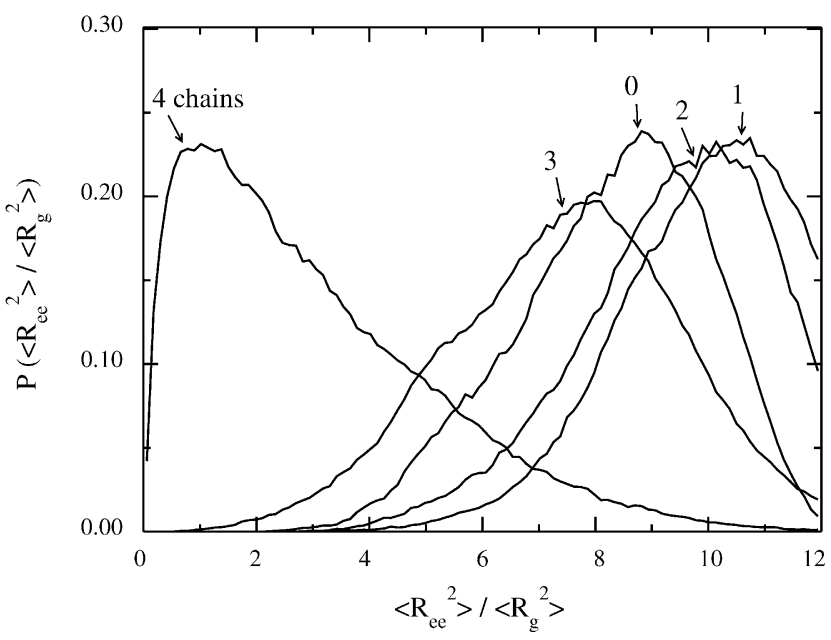

Fig. 8. Shape distribution functions for different numbers of positive chains (systems $(1-4 p+30)$ ). Also included, for reference, is the shape distribution of the polyanion in the presence of its small counter-ions. Curves are labelled according to the number of polycation chains. From Ref. [98]. 


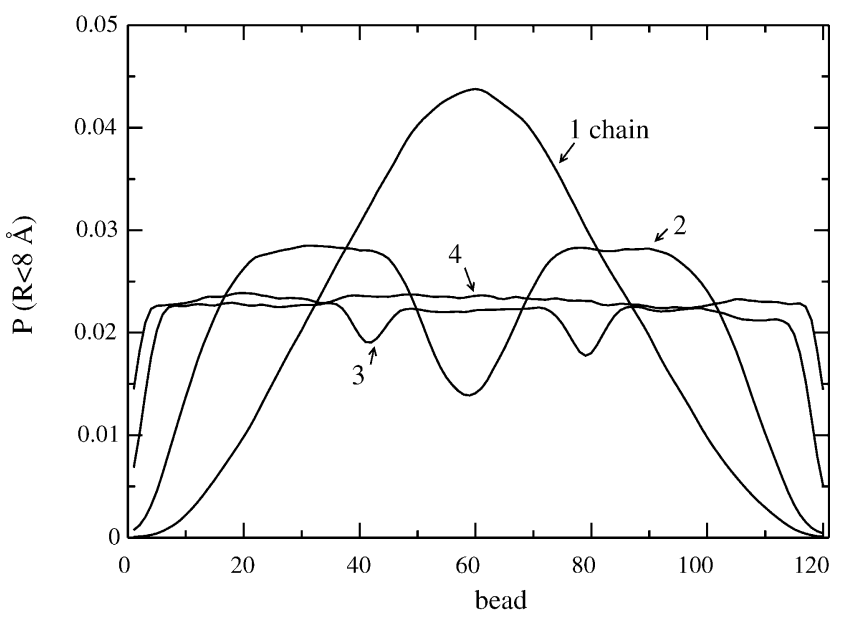

Fig. 9. Probability of finding a polycation bead at a distance below $8 \AA$ from each negative polyelectrolyte segment for the systems of Fig. 8. From Ref. [98].
A similar picture is found for three chains, now with two minima in the probability. When $\beta=1$ is attained, no preferential positioning can be discerned. A direct observation of the illustrative snapshots of Fig. 10 further indicates that a slight folding characterizes the part of the chain in contact with the polycation (for $\beta<1$ ).

If we relate the mobility of the polycations along the negative chain, e.g. expressed in terms of the width of the contact between positive and negative beads, with the degree of compaction, we note a significant pattern. When only one chain is present, it covers a substantial length of the polyanion, the latter being characterized by stretched conformations. When two chains are present, they repel each other but, typically, each still covers more than $35 \%$ of the polyanion, again giving rise to unfolded shapes. A more drastic effect occurs for three chains, additionally reducing mobility and slightly increasing the degree of compaction. Finally, when four polycations are considered, definite compaction is achieved and the system is characterized by the lowest mobility.



(b)
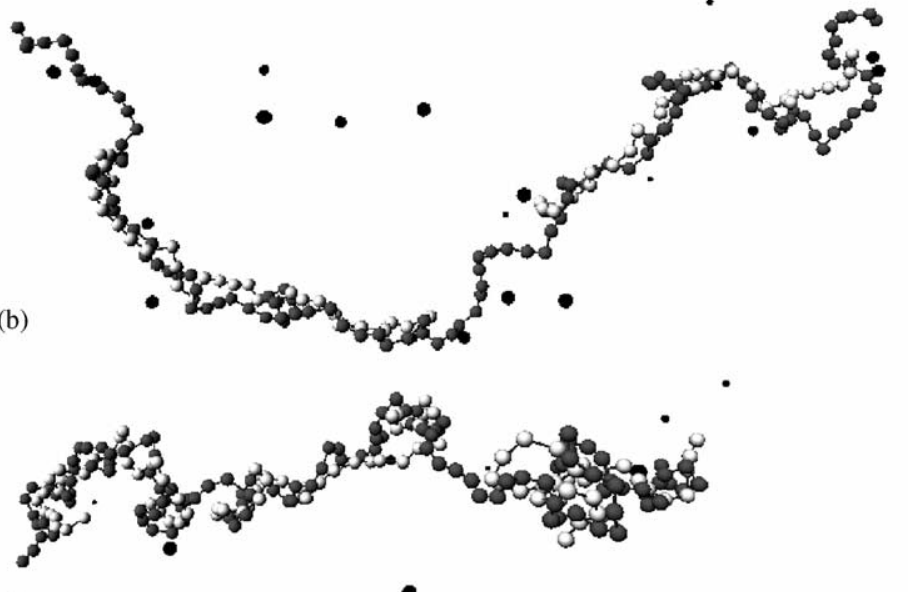

$\bullet$

(c)

(d)

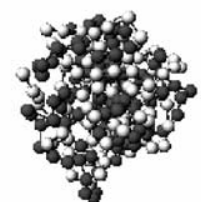

Fig. 10. Configurations for different numbers of positive chains interacting with the negative one (systems $(1-4 p+30)$ ). Key: (a) 1 , (b) 2 , (c) 3 , and (d) 4 polycation chains. From Ref. [98]. 
For simplicity, some of the systems treated in this work did not contain counter-ions. These have been included only for $\beta \neq 1$ to achieve electroneutrality. The compaction action took place in most of the considered systems in the absence of counter-ions. For system $(4 p+30)$ we have duplicated the calculations, now in presence of counter-ions and co-ions, so as to check the effect of counter-ion release in the degree of compaction. Results in this modified system have shown conformation indicators very similar to those obtained from the original one. At least for these model systems, it is apparent that the electrostatic interactions clearly surmount counter-ion release effects. We believe that when the compacting agent approaches the chain, this release takes place and entropically favours substitution of the counter-ion cloud by more strongly bound arrays of charges. However, in these systems, it was shown that the electrostatic interaction was sufficient to counterbalance the entropy loss resulting from fixation of the longer polycations and reduced polyanion volume.

We have studied the action of polycations as compacting agents of a medium size anionic polymer. Although some differences may be found between typical experimental systems involving DNA and the model system in this work (persistence length of the polyelectrolyte, concentration, etc.) we believe that our observations can be extended to the former. Results have shown that the degree of compaction is determined by the size of the positive chains and their number. The role of electrostatic interactions is paramount in the compaction process and, an increase in the number of molecules of the compacting agent or in the number of charges on each molecule causes sudden collapse of the polyanion molecule. Compaction is associated with polycations promoting bridging between different sites in the negatively charged polymer. Another interesting finding is that the interaction for polycation/polyanion charge ratios significantly below 1 produces only a small degree of intrachain segregation, allowing for significant translation freedom of the agent along the chain.

An important conclusion is that monovalent counter-ions play only a minor role in the folding process.

\section{DNA interactions with catanionic mixtures: DNA-surfactant complexes decompaction by anionic amphiphiles}

The interest in liposomes, and their interactions with DNA, have grown since they appeared as promising carriers for gene delivery and transfection [53]. Despite the numerous studies of DNA interaction with liposomes, constituted by cationic and neutral lipids, there has been very little attention on the DNA behaviour in the presence of mixtures of two oppositely charged surfactants. Nevertheless these systems present certain interest for the pharmaceutical applications since variations on the mixing ratios between oppositely charged amphiphiles may strongly affect the linear dimensions and the charge of individual DNA and, con- sequently, influence the process of a transmembrane DNA delivery.

Mixtures of cationic and anionic surfactants are known as catanionic mixtures [87] and have, due to their rich phase behaviour and interfacial properties, been the subject of many studies (for reviews see Refs. [87,88]). By varying the mixture composition, i.e., total surfactant concentration and mixing ratios, one can obtain aggregates with different geometries, ranging from spherical, to cylindrical and planar. The most notable structures these systems form are vesicles, and catanionic vesicles are believed to be thermodynamically stable; in fact they are formed spontaneously and reversibly, and remain stable for a long period of time [89].

The system used in this work is the aqueous mixture of CTAB and sodium octylsulfate (SOS), studied by Kaler and coworkers [90,91], and FM was used to follow the conformational changes of long DNA in the presence of the catanionic vesicles.

\subsection{DNA behaviour in the presence of negatively charged catanionic mixtures}

Upon addition of DNA stock solution to the samples containing negatively charged vesicles no change in the conformational behaviour of individual DNA molecules was detected. This result seems plausible, taking into account the strong repulsive interactions between the similarly charged objects, however, since the vesicles contain individual cationic surfactants, these could be extracted and interact with DNA. Since no globular DNA's were found in solution we decided to check on the stability of the DNA globules formed in the presence cationic surfactant upon the addition of anionic surfactant.

We started by compacting DNA with CTAB. For the chosen concentration of surfactant, $3.16 \times 10^{-4} \mathrm{M}$, we could observe, by fluorescence microscopy, that all DNA molecules were in the compacted state, according to the results presented above. Then the anionic surfactant, SOS, was added stepwise. No visible effect was noted while the concentration was below $3.16 \times 10^{-4} \mathrm{M}$. When the SOS concentration reached $3.8 \times 10^{-4} \mathrm{M}$, unfolded DNA coils were found in the sample solution together with DNA-CTAB globules. A further increase in the anionic surfactant concentration resulted in the complete unfolding of DNA-CTAB complexes at $[\mathrm{SOS}]=6.31 \times 10^{-3} \mathrm{M}$. All DNA molecules were in the coil conformation (Fig. 11).

\subsection{Dependence on the anionic surfactant chain length}

We investigated also the influence of the anionic surfactant chain length as well as the role of the cationic amphiphile hydrophobicity on the dissolution of the DNA-cationic surfactant complexes.

We observed that for a longer chained anionic surfactant, sodium dodecylsulphate (SDS), a smaller amount of surfactant was required to induce decompaction. As observed in 


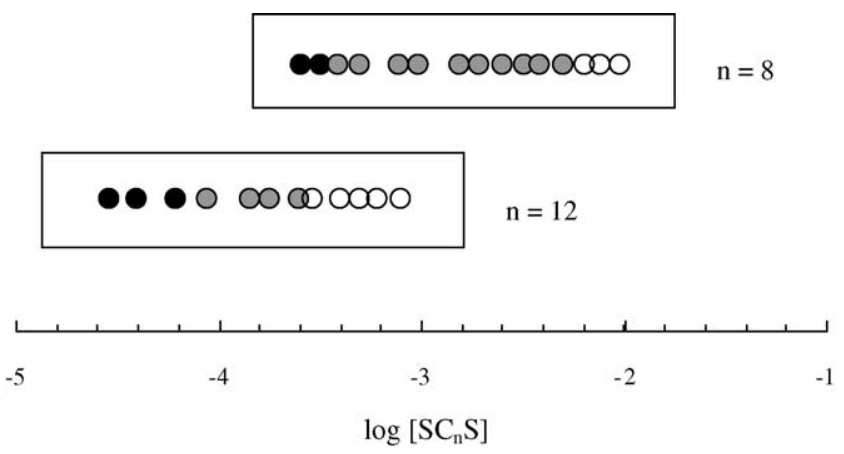

Fig. 11. Dependence of the conformational behaviour of single T4DNA molecules, $0.5 \mu \mathrm{M}$ in aqueous buffer solution and a constant DTAB concentration of $3.16 \times 10^{-4} \mathrm{M}$, on the stepwise addition of SDS and SOS. Filled circles correspond to the globular DNA conformation, and shaded circles to the coexistence between elongated coils and compacted DNA molecules, whereas open circles correspond to the extended conformation of DNA. $T=25^{\circ} \mathrm{C}$. From Ref. [13].

Fig. 11 a concentration of $8.71 \times 10^{-5} \mathrm{M}$ was enough to start the DNA unfolding and for $2.88 \times 10^{-4} \mathrm{M}$, below charge neutralization, all DNA molecules were in the coil conformation.

This is easy to understand from the chain length dependence of surfactant self-assembly. On adding the anionic surfactant to the solution of DNA and cationic surfactant, above a certain concentration it will associate and form mixed selfassemblies with the oppositely charged amphiphile and release DNA back into the solution as a coil. The onset of this association can be defined in terms of a critical micellar concentration for the mixture of the two surfactants $\left(\mathrm{CMC}_{\mathrm{mixt}}\right)$. Since SDS is more hydrophobic than SOS, the $\mathrm{CMC}_{\text {mixt }}$ for that surfactant will be smaller than the shorter chained one.

\subsection{Dependence on the cationic surfactant chain length}

As mentioned we also performed experiments to determine the dependence of DNA decompaction on the chain length of the compacting amphiphile, CTAB, TTAB, and DTAB. Following the same procedure as above we started by compacting DNA with $3.16 \times 10^{-4} \mathrm{M}$ of CTAB, for example, and added the anionic amphiphile stepwise. Contrary to the experiments varying the anionic surfactant we found no variation on changing the cationic surfactant chain length (Fig. 12), that is, the amount of negatively charged surfactant necessary to unfold DNA is independent of the hydrophobicity of the compacting amphiphile.

This can be explained as follows. Above a certain $\mathrm{CMC}_{\text {mixt }}$, dependent on the anionic surfactant chain length, two types of structures can be formed in solution, DNA-cationic surfactant $\left(\mathrm{DNA}^{+} \mathrm{S}^{+}\right)$globules and cationic-anionic surfactant aggregates $\left(\mathrm{S}^{+}-\mathrm{S}^{-}\right)$. In the case of CTAB and SOS, for example, when we observe an unfolding of DNA molecules, there is a dissociation of DNA-CTAB globules, with CTAB molecules leaving DNA-CTAB complexes and transferring to CTAB-SOS aggregates. Since this

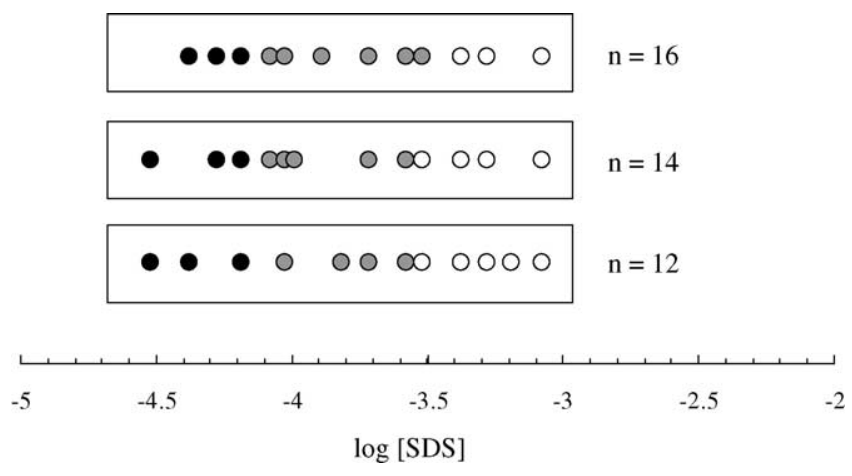

Fig. 12. Dependence of the conformational behaviour of single DNA molecules, $0.5 \mu \mathrm{M}$ in aqueous buffer solution at constant concentrations of cationic surfactants CTAB, TTAB and DTAB, $3.16 \times 10^{-4} \mathrm{M}$, on the total SDS concentration. Symbols are the same as in Fig. $11 . T=25^{\circ} \mathrm{C}$. From Ref. [13].

"transfer" of molecules is occurring between two different surfactant aggregates, it will not depend on the surfactant chain length, or alternatively expressed there is a cancellation in the alkyl chain length effects in forming the two types of aggregates.

\subsection{Surfactant aggregate structures}

We can note from the above results that the interaction between the oppositely charged surfactants is stronger than the one between DNA and cationic amphiphiles. A question that remains is then, what type of aggregates will the surfactants form. To answer this we performed some cryogenic transmission electron microscopy (cryo-TEM) experiments, since this technique allows a good visualization of nm-size objects.

We found structures of crystalline appearance in most of the samples (Fig. 13a). This is not unexpected since both surfactants had the same chain length and approximately the same concentrations. It is known that most catanionic systems precipitate at equimolar concentrations even at very high water contents [88].

Along with the precipitate we observed, under other conditions, the formation of vesicles (Fig. 13b). The formation and stability of vesicles are dependent on the surfactant chain type and length. Thus, vesicle regions are usually larger when one of the amphiphiles has a double chain or when two single chained surfactants have asymmetric chains, which has been attributed to optimal packing conditions [92]. The presence of vesicles in the sample is then due to a large excess of the anionic surfactant and an asymmetry in the surfactant chain lengths, SOS versus DTAB $[89,93]$.

These findings led us to enquire whether it was possible to predict the structures formed by the surfactant mixture, subsequent to DNA compaction and decompaction, if we had the knowledge of the phase diagram of the mixture. In particular we investigated this issue by additional cryo-TEM experiments. We chose a point in the CTAB/SOS/water phase diagram [90,91], with $9.88 \mathrm{mM}$ of CTAB and $43.00 \mathrm{mM}$ of SOS, 


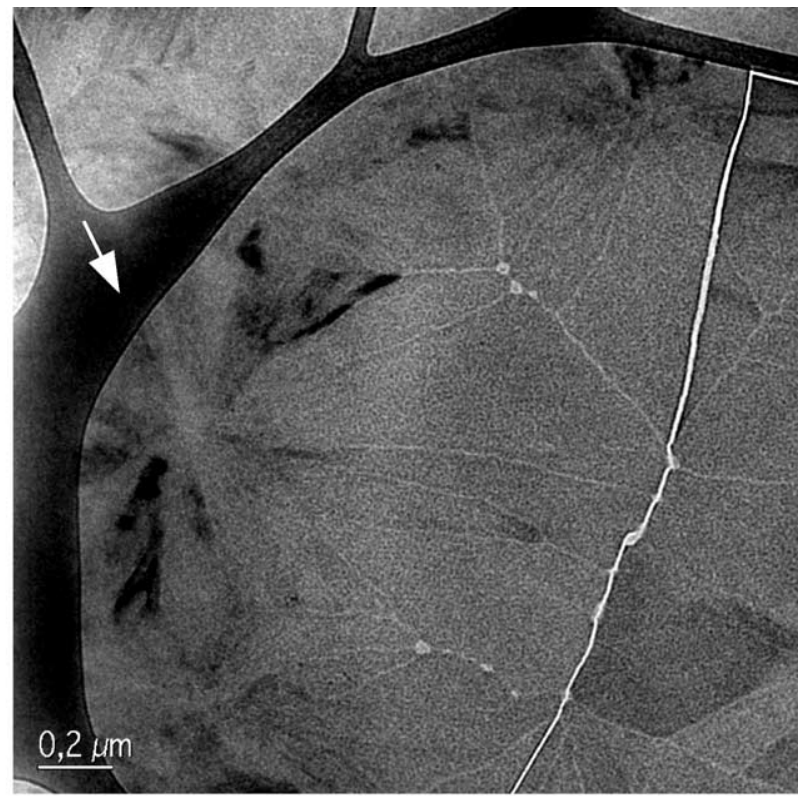

(a)

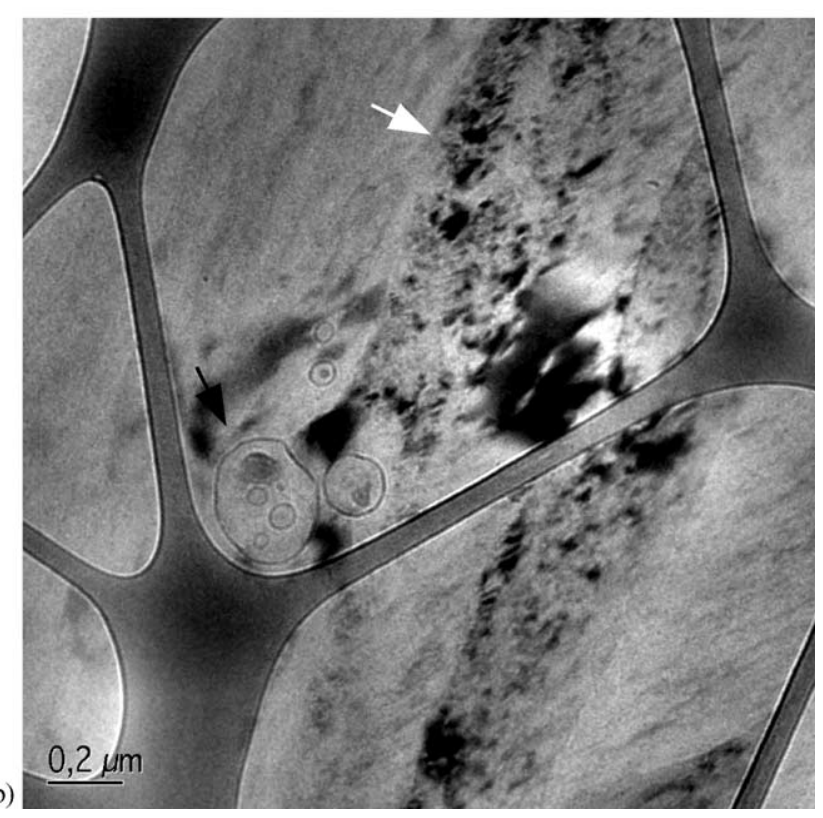

Fig. 13. Cryo-TEM images of the surfactant structures formed subsequent to the DNA compaction with DTAB and addition of the anionic amphiphile SDS $\left(0.5 \mu \mathrm{M}\right.$ of T4DNA in buffer solution; $3.16 \times 10^{-4} \mathrm{M}$ of DTAB). In (a) $[\mathrm{SDS}]=8.13 \times 10^{-4} \mathrm{M}$ and in (b) $[\mathrm{SOS}]=7.94 \times 10^{-3} \mathrm{M}$. Crystals are denoted by white arrows and vesicles by black arrows. Redrawn from Ref. [13].

which corresponds to the region of negatively charged vesicles. This region was selected since vesicles are self-assembly structures easy to observe and recognize as well as one of the most interesting for application purposes.

In the solution, as expected, we observed only the presence of somewhat polydisperse unilamellar vesicles with sizes ranging from 20 to $100 \mathrm{~nm}$ (Fig. 14a).

A sample with the same surfactant concentrations was prepared using the normal FM procedure: we observed in the microscope that in a DNA buffer solution with $9.88 \mathrm{mM}$ (a)

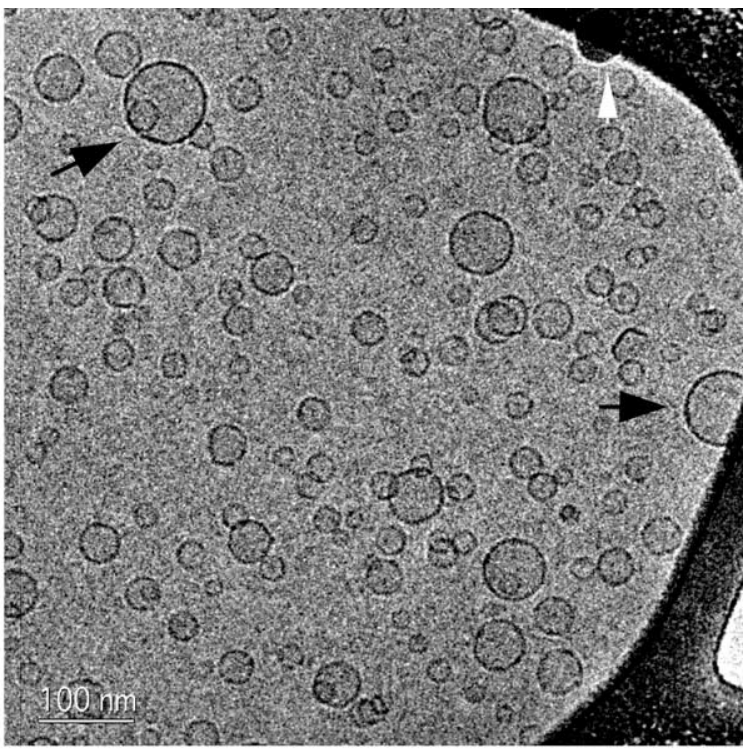

(b)

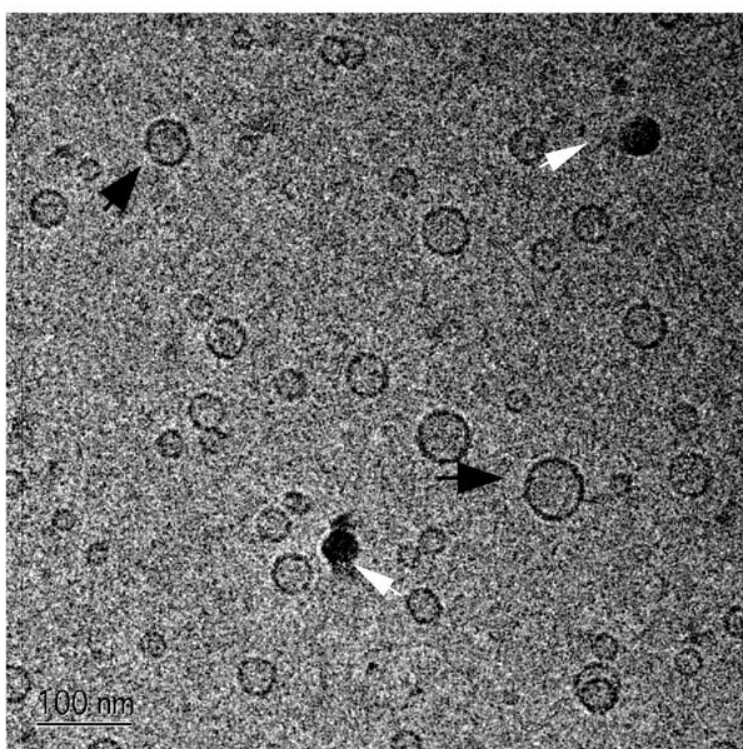

Fig. 14. (a) Cryo-TEM images of an aqueous solution of SOS, $4.3 \times 10^{-2} \mathrm{M}$, and CTAB, $9.88 \times 10^{-3} \mathrm{M}$ in the absence (a) and presence (b) of DNA $5.0 \times 10^{-7}$ M. (a), (b), and (c) unilamellar vesicles. Black arrows denote vesicles, whereas the white ones indicate crystallized water. Redrawn from Ref. [13].

of CTAB, all DNA molecules presented a compact conformation; with the addition of the anionic surfactant, SOS at $43.00 \mathrm{mM}$, only coils were present in solution. These concentrations of amphiphiles were enough to induce compaction and decompaction of the DNA molecules. Observing then the surfactant aggregates, by cryo-TEM, we realized that there was no visible difference between this sample and the previous one, prepared by mixing surfactants alone (Fig. 14b versus a). Again, only small unilamellar vesicles were present in solution. Note that these findings provide support for thermodynamic stability of the vesicles, still a matter somewhat controversial. 
The ability of controlling the structures formed by the surfactants is significant and promising for a number of applications. For example, when using cationic surfactants to compact DNA for purification purposes, the addition of an amount of anionic surfactant would both release DNA back into solution and form a precipitate with the oppositely charged surfactant. This would enable a simple and efficient separation of DNA from the surfactants in solution. From another point of view, for an eventual application of these types of systems to gene therapy, the presence of crystals would be catastrophic for the cells.

All of this discussion was conducted by focusing on the amphiphile aggregates and the DNA conformational behaviour. There is no indication that the DNA molecule undergoes denaturation and/or degradation during the compaction and release process. It has been reported [12] that the DNA released from cationic lipid complexes by the addition of anionic additives was in its native B-form conformation.

It was accordingly observed that anionic amphiphiles can be used to unfold and release DNA previously compacted by cationic surfactants. We found that by using anionic surfactants with longer chains the decompaction of DNA is more efficient, while it does not depend on the hydrophobicity of the compacting amphiphile. Our results also suggest that one can predict and control the aggregates the oppositely charged surfactants will form, subsequent to DNA folding and release, simply from the added amounts of the amphiphiles and knowledge of their phase map.

\section{DNA interactions with catanionic mixtures: DNA compaction and adsorption onto positively charged vesicles}

Contrary to the results obtained for anionic-rich catanionic mixtures, the addition of oppositely charged mixtures to DNA solution leads to the collapse of individual DNA chains. It was noticed that the diffusion rate of the DNA globules was substantially lower than for DNA globules formed in the presence of simple cationic surfactant. This apparent increase in size was due to the adsorption of the DNA globules onto the positively charged vesicles as supported by the results of optical microscopy observations (Fig. 15). In this particular experiment we illuminated the samples with both UV- and visible light simultaneously. Therefore the observation of both fluorescently labelled DNA and large catanionic vesicles was possible. The vesicles in the samples were quite polydisperse and some big enough to allow for their observation with the use of the optical microscope.

In conclusion, our results suggest that the positively charged catanionic vesicles may be successfully applied to induce the folding transition of a large single DNA and adsorption of the DNA globules onto the surface of the vesicles. Recharging the vesicles through change of the molar ratio between cationic and anionic lipids in solution leads to DNA unfolding and release to the bulk solution.

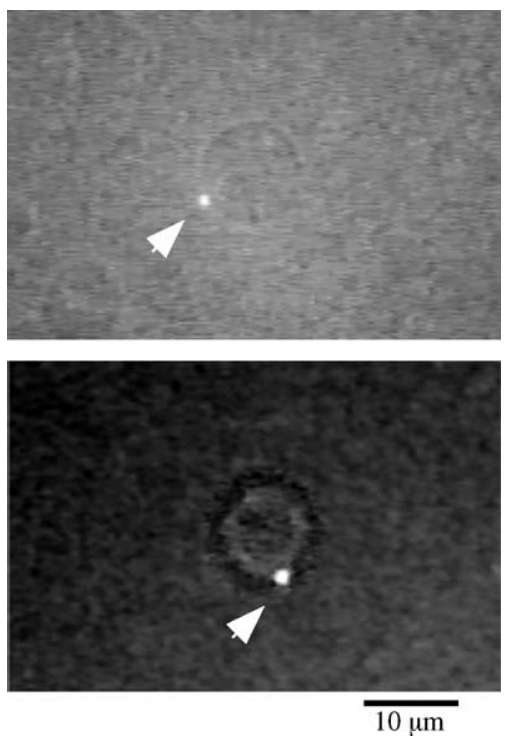

Fig. 15. Optical microscopy images of single T4DNA globules adsorbed onto the surface of positively charged catanionic vesicles $([\mathrm{CTAB}]=17.27 \mathrm{mM}$ and $[\mathrm{SOS}]=15.00 \mathrm{mM})$. The DNA concentration is equal to $4.54 \mathrm{nM}$ in phosphate groups. Arrowheads indicate single DNA globules. From Ref. [94].

\section{Interaction between polyelectrolytes and catanionic surfaces}

The interaction of polyelectrolytes and surfaces bearing charges is a phenomenon of paramount importance in a variety of biological, technological and industrial processes. Differences in the characteristics of the surfaces (curvature, charge, charge density) induce different behaviours in the binding of the polyelectrolyte. For highly charged surfaces with sign opposite to that of the polyelectrolyte, binding is so strong that the dynamics of the polyelectrolyte backbone can be studied in two dimensions [64], if other particles in the solution are not taken into consideration. In some cases surfaces may display both negative and positive charges. In these cases, the heterogeneity of the charge distribution also has some impact in the behaviour of the adsorbed polyelectrolyte [68].

As we have seen a significant amount of experimental work in our group has been focused on the interaction between catanionic vesicles and DNA [94,58]. The interaction caused both a certain degree of compaction in the polymer backbone, as observed through fluorescence microscopy, and a change in the shape in the vesicles, consistent with the gelification of the latter.

These experimental results have prompted a Monte Carlo study [95] of a model system in which mobile charges are confined to a layer, with a thickness corresponding to the diameter of the surface charges. They are thus able to move in the $x y$ plane, but are fixed along the $z$-axis. A "negative bead and spring" polyelectrolyte with a varying degree of rigidity interacts with this surface. Surface and/or polylectrolyte monovalent counter-ions are introduced so as to render the 


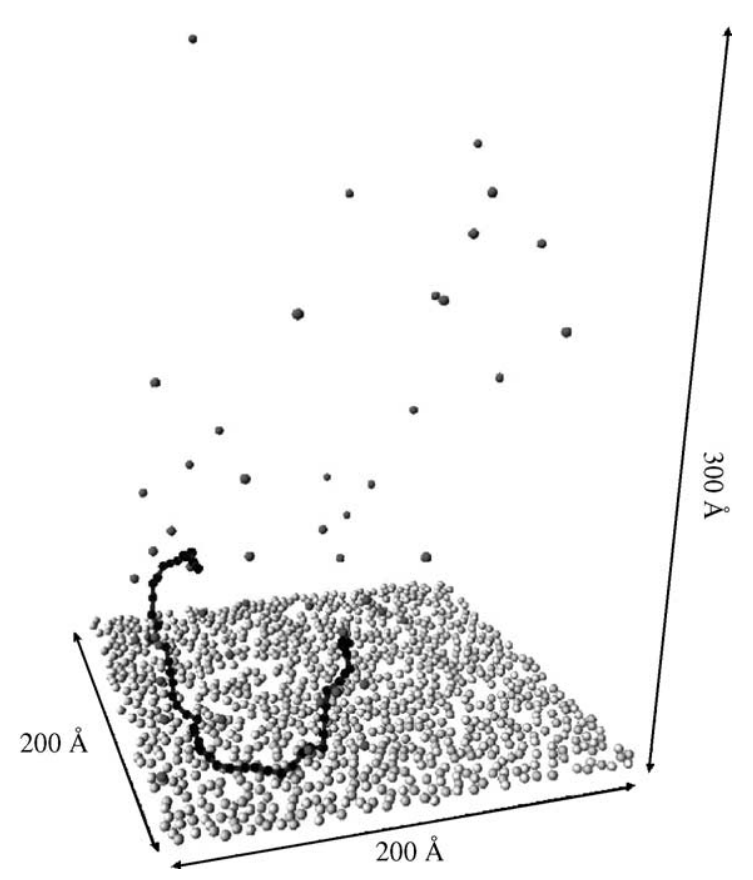

Fig. 16. Model for the polyelectrolyte-catanionic membrane interaction, including dimensions of simulation box. All particles, represented as hardspheres, have a radius $R=2 \AA$.

system electroneutral. We have used periodic boundary conditions in the plane of the surface and hard walls exactly below the surface charges, and at a distance of $300 \AA$ from this surface. The polyelectrolyte beads may thus be found above or within the layer, but not below. The illustration in Fig. 16 summarises the model setup.

The number of particles confined to the surface was kept constant (1000) in our studies, but the overall surface charge was varied from $+1000 e$ to $-200 e$, where $e$ is the elementary charge. The polyelectrolyte length was also varied (25 and 50 beads) and so was its intrinsic stiffness.

The mobile charges in the surface react to the presence of the polyelectrolyte and a globally neutral membrane with the above characteristics determines a drastic degree of adsorption. The $z$-axis density function of Fig. 17 indicates that the 50 bead long polyelectrolyte resides essentially in close contact with the surface. The polyelectrolyte counter-ions are also in the vicinity of the surface, i.e., travel in association with the polyion. The presence of the polyion polarises the membrane. We have divided the $200 \AA \times 200 \AA$ surface in 100 squares and plotted the probability density function for the charge of these squares in Fig. 18. For a neutral membrane in the absence of the polyelectrolyte this distribution is centred in zero and decays rapidly for both negative and positive values. On average, 10 charges are to be found in each square, so the maximum net charge would amount to ca. $+10 e$ or $-10 e$. It can be seen from that figure that the probability of finding squares with an absolute value of the charge exceeding $5 e$ is very close to zero. The presence of the polyelectrolyte causes a visible increase in the number

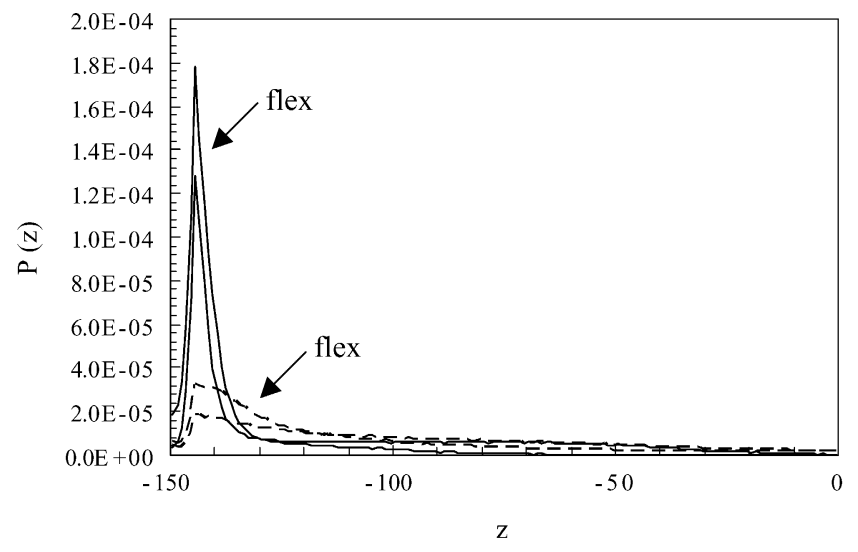

Fig. 17. Density of particles along the $z$-axis, i.e., the direction normal to the surface. The solid line corresponds to polyelectrolyte (the most flexible is indicated by arrows), and the dashed-line to the respective counter-ions. From Ref. [95].

of more highly charged positive regions (from $+2.5 e$ to $+7 e$ ), while decreasing the number of squares close to electroneutrality and, again, increasing the number of negative, but less charged ones $(-1 e$ to $-5 e)$. Although this effect may appear less significant, it should be recalled that we are observing the effect of a polyelectrolyte described by 50 positive monovalent beads in a surface comprising 500 positive and 500 negative charges.

The above observations indicate that the adsorption behaviour of the polyelectrolyte must be, to a significant degree, influenced by the response given by the membrane to its presence. It can be seen in Fig. 19 that the number of adsorbed beads increases drastically with an increasing flexibility of the polyelectrolyte. In fact, calculations involving

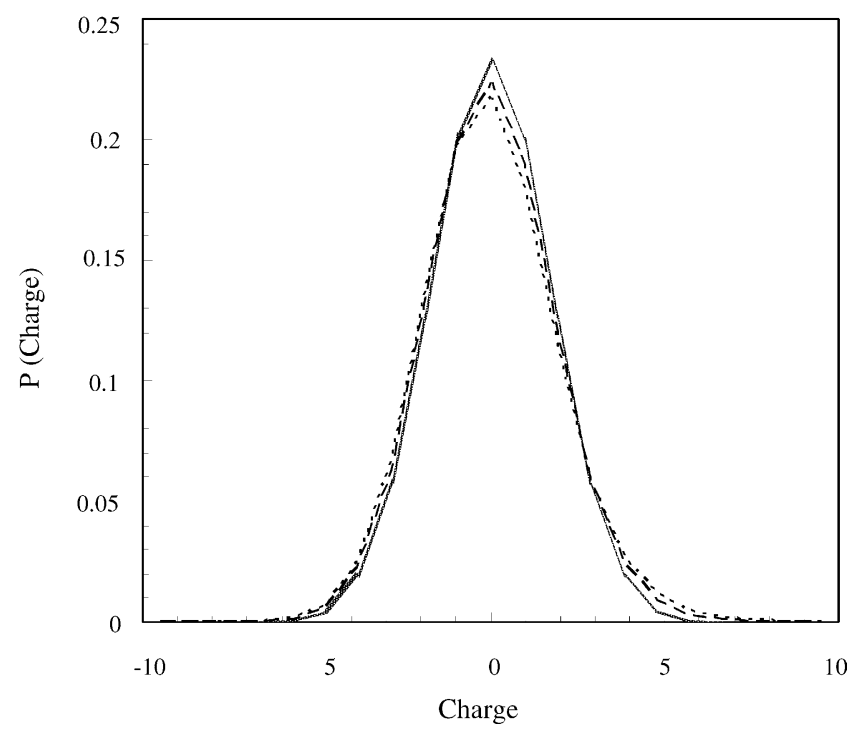

Fig. 18. Probability distribution of charge in the catanionic surface, corresponding to a subdivision in square areas of $20 \AA \times 20 \AA$. The solid line corresponds to the surface without polymer, the dashed-line to the system with the flexible polyelectrolyte ( 50 beads) and the dotted-line to the semiflexible one. From Ref. [95]. 


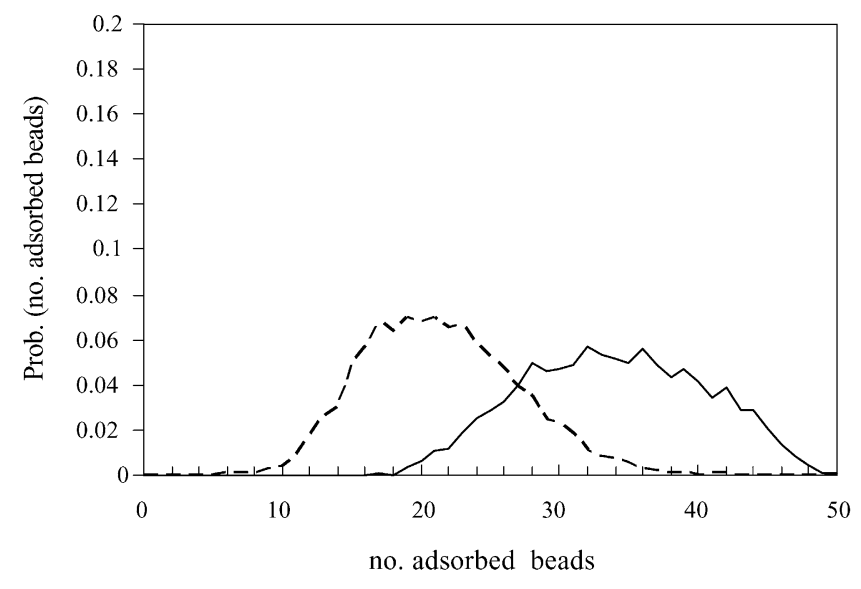

Fig. 19. Probability of number of adsorbed beads. The solid line corresponds to the flexible polyelectrolyte (50 beads) and the dashed-line to the corresponding semi-flexible polyelectrolyte.

three values for the imposed intrinsic stiffness have shown that this variation is not monotonic. In ascending order of the average number of adsorbed beads we have values for the angular sequential three-bead angular force constant of 0 , $3.4 \mathrm{E}-24$ and $1.0 \mathrm{E}-20 \mathrm{~J} \mathrm{deg}^{-2}$. The usual picture in which a more flexible polymer faces a larger entropic resistance to adsorption, related to the decrease in the conformational degrees of freedom, is not followed. Apparently, the concentration of opposite charges in the surface of the membrane is dictated partially by the conformation in bulk, but smaller degrees of polarisation (compatible with more stretched backbones) may also favour adsorption. In other words, a flexible polyelectrolyte concentrates negative charge and induces more positive charge in a localised zone of membrane, thus adsorbing easily. However, for more stretched ones, this concentration is not necessary, and it may adsorb without a large degree of disruption in the random charge distribution.

We have also observed that a smaller polyion ( 25 beads) typically adsorbs more completely than the larger one (50 beads). This indicates that the polarisation in the membrane becomes less facilitated as the degree of disruption increases.

A globally negative membrane (comprising 505 negative particles and 495 positive ones) still induces a significant degree of adsorption, but for a total charge of -50 , the polyion consisting of 50 beads tends to stay in the bulk.

\section{Concluding remarks}

Our investigation of the interactions between DNA and alkyltrimethylammonium bromide surfactants of various chain lengths has been reviewed.

The systems were studied in the dilute regime, for DNA concentrations of $0.5 \mu \mathrm{M}$, by fluorescence microscopy and conformational maps were drawn illustrating the surfactant hydrophobic interactions present in the compaction; a larger concentration of DTAB, the shorter chained surfactant, is needed to observe the compaction of DNA molecules.

The discrete phase transition from DNA coils to globules observed by FM in the presence of cationic surfactants was also observed by dynamic light scattering, where the size of the complexes could be discerned more precisely.

A simulation study on the compaction of a medium size anionic polymer by polycations was presented. The degree of compaction was found to be determined by the size of the polycations and their number. The role of electrostatic interactions is crucial in the compaction process since an increase in the number of charges per chain or in the number of chains of the compacting agent leads to a sudden collapse of the polyanion molecule, which is in agreement with the fluorescence microscopy observations.

It was found that the compaction is associated with polycations promoting bridging between different parts of the negatively charged polymer. The interaction for polycation/polyanion charge ratios below one produces only a small degree of intra-chain segregation allowing a significant translational freedom of the polycations along the longer chain, which suggests that a loss of this translational entropy of the compaction agent is required for the collapse of the polyanion. This is in agreement with the experimental results. Finally, an important conclusion was that the counter-ions play only a minor role in the folding process.

We found that DNA does not interact with negatively charged catanionic vesicles even though they carry some positively charged molecules. However it was found to compact and adsorb in the form of a globule onto the surface of positively charged vesicles. We also showed that it is possible to dissociate DNA-cationic surfactant complexes by the addition of an oppositely charged amphiphile. The decompaction was followed and it was found that by using anionic surfactants with a longer alkyl chain the decompaction was more efficient, that is, less surfactant was required for the dissolution of the DNA-surfactant complexes. However, it does not depend on the hydrophobicity of the cationic surfactant. The decompaction occurs due to the formation of mixed surfactant aggregates. The results presented also suggest that it is possible to predict the structures that the surfactant will form, subsequent to DNA folding and release, from the knowledge of their phase map and, consequently, from the added amounts of both amphiphiles.

These observations can be interesting from an application viewpoint, since the balance between compacted/decompacted DNA molecules with different concentrations of oppositely charged lipids, the aggregates of which can be controlled, can be of interest to applications like DNA purification or controlled DNA delivery.

Finally Monte Carlo simulations were performed on the adsorption of a polyelectrolyte on catanionic surfaces. The model system consisted of a negatively charged polymer with a varying degree of rigidity and length, and a surface consisting of mobile charges confined to a layer able to move only in the $x y$ plane but fixed along the $z$-axis. As predicted the mobile 
charges in the surface react to the presence of the polyelectrolyte enabling a high degree of adsorption even though the membrane was globally neutral. Our observations indicate that the adsorption behaviour of the polyelectrolyte is influenced by the response given by the membrane to its presence. One interesting observation was that the number of adsorbed beads increases drastically with the increase of flexibility of the polymer. In fact, calculations involving polymers with three different intrinsic stiffness showed that the variation is non-monotonic. We also observed that a smaller polyanion typically adsorbs more completely than the larges one, which indicates that the polarisation of the membrane becomes less facilitated the more it is "disturbed".

\section{Acknowledgments}

This work was supported by grants from Praxis XXI (PRAXIS/BD/21227/99), the Fundacao para a Ciencia e Tecnologia (FCT) (POCTI/QUI/45344/2002, and POCTI/QUI/45331/2002). The dynamic light scattering work is made in collaboration with Otto Glatter and Josef Innerlohinger, Graz, and the simulation work with the collaboration and support from Per Linse, Lund.

\section{References}

[1] E. Wagner, M. Cotten, R. Foisner, M.L. Birnstiel, Proc. Natl. Acad. Sci. U.S.A. 88 (1991) 4255.

[2] M. Ogris, P. Steinlein, M. Kursa, R. Mechtler, E. Wagner, Gene Therapy 5 (1998) 1425.

[3] T. Kimura, T. Yamaoka, R. Iwase, A. Murakami, Macromol. Biosci. 2 (2002) 437.

[4] K. Yoshikawa, Adv. Drug Del. Rev. 52 (2002) 235.

[5] M. Köping-Höggard, Y.S. Mel'nikova, K.M. Vårum, B. Lindman, P. Artursson, J. Gene Med. 5 (2003) 130.

[6] D.D. Lasic, Liposomes in Gene Delivery, CRC Press, Boca Raton, 1997.

[7] P. Pinnaguwage, L. Huang, Biochemistry 31 (1992) 2850.

[8] T. Kimura, R. Yamaoka, R. Iwase, A. Murakami, Macromol. Biosci. 2 (2002) 437.

[9] W.M. Bertling, M. Gareis, V. Paspaleeva, A. Zimmer, J. Kreuter, E. Nurnberg, P. Harrer, Biotechnol. Appl. Biochem. 13 (1991) 390.

[10] K. Crook, G. McLachlan, D.J. Porteous, Gene Therapy 3 (1996) 834.

[11] X. Gao, L. Huang, Biochemistry 35 (1996) 1027.

[12] S. Bhattacharya, S.S. Mandal, Biochemistry 37 (1998) 7764.

[13] R. Dias, B. Lindman, M. Miguel, J. Phys. Chem. B 106 (2002) 12608.

[14] O. Zelphati, F.C. Szoka, Proc. Natl. Acad. Sci. 93 (1996) 11493.

[15] A. Trewavas, Anal. Biochem. 21 (1967) 324.

[16] K. Hayakawa, J. Santerre, J. Kwak, J. Biophys. Chem. 17 (1983) 175.

[17] K. Shirahama, K. Takashima, N. Takisawa, Bull. Chem. Soc. Jpn. 60 (1987) 43.

[18] A. Gorelov, E. Kudryashov, J.-C. Jacquier, D. McLoughlin, K. Dawson, Phys. A 249 (1998) 216.

[19] S.M. Mel'nikov, V.G. Sergeyev, K. Yoshikawa, in: S.G. Pandalai (Ed.), Recent Research Developments in Chemical Sciences, Trivandrum, 1997, p. 69.
[20] S. Bhattacharya, S.S. Mandal, Biochim. Biophys. Acta 1323 (1997) 29.

[21] W.J. Jin, Y.S. Wei, C.S. Liu, G.L. Shen, R.Q. Yu, Spectrochim. Acta A 53 (1997) 2701.

[22] V.A. Izumrudov, M.V. Zhiryakova, A.A. Goulko, Langmuir 18 (2002) 10348.

[23] R. Dias, S. Mel'nikov, B. Lindman, M. Miguel, Langmuir 16 (2000) 9577.

[24] S. Guillot, D. McLoughlin, N. Jain, M. Delsanti, D. Langevin, J. Phys.: Condens. Mat. 15 (2003) S219.

[25] R. Ghirlando, E.J. Wachtel, T. Arad, A. Minsky, Biochemistry 31 (1992) 7110

[26] S.M. Mel'nikov, V. Sergeyev, K. Yoshikawa, H. Takahashi, I. Hatta, J. Chem. Phys. 107 (1997) 6917.

[27] D. McLoughlin, Ph.D. Thesis, University College, Dublin, 2000.

[28] P. Smith, R.M. Lynden-Bell, W. Smith, Phys. Chem. Chem. Phys. 2 (2000) 1305

[29] C. Leal, L. Wadsö, G. Olofsson, M. Miguel, H. Wennerström, J. Phys. Chem. B 108 (2004) 3044.

[30] S.M. Mel'nikov, M.O. Khan, B. Lindman, B. Jönsson, J. Am. Chem. Soc. 121 (1999) 1130.

[31] K. Minagawa, Y. Matsuzawa, K. Yoshikawa, A.R. Khokhlov, M. Doi, Biopolymers 34 (1994) 555.

[32] K. Yoshikawa, M. Takahashi, V.V. Vasilevskaya, A.R. Khokhlov, Phys. Rev. Lett. 76 (1996) 3029.

[33] K. Yoshikawa, S. Kidoaki, M. Takahashi, V.V. Vasilevskaya, A.R Khokhlov, Ber. Bunsenges. Phys. Chem. 100 (1996) 876.

[34] Y. Yamasaki, Yoshikawa, J. Am. Chem. Soc. 119 (1997) 10573.

[35] L. Guldbrand, B. Jönsson, H. Wennerström, P. Linse, J. Chem. Phys. 80 (1984) 2221.

[36] M.O. Khan, Ph.D. Thesis, University of Lund, 2001.

[37] Y. Matsuzawa, Y. Yonezawaand, K. Yoshikawa, Biochem. Biophys. Res. Commun. 225 (1996) 796.

[38] D.L. Nelson, M.M. Cox, Lenhinger Principles of Biochemistry, 3rd ed., Wroth Publishers, New York, 2000.

[39] L. Stryer, Biochemistry, 4th ed., W.H. Freeman Company, New York, 1995.

[40] P.J. Horn, C.L. Peterson, Science 297 (2002) 1824.

[41] H. Tabor, Biochem. Biophys. Res. Commun. 3 (1960) 382.

[42] H. Tabor, Biochemistry (Wash.) 1 (1962) 496.

[43] W.H. Braunlin, T.J. Strick, M.T. Record, Biopolymers 21 (1982) 1301.

[44] L.C. Gosule, J.A. Schellman, Nature 259 (1976) 333.

[45] D. Porschke, Biochemistry 23 (1984) 4821.

[46] S. Razin, R. Rozansky, Arch. Biochem. Biophys. 81 (1959) 36.

[47] E. Raspaud, M.O. Cruz, J.-L. Shikorav, F. Livolant, Biophys. J. 74 (1998) 381.

[48] N. Korolev, A.P. Lyubartsev, L. Nordenskiöld, A. Laaksonen, J. Mol. Biol. 308 (2001) 907.

[49] K. Minagawa, Y. Matsuzawa, K. Yoshikawa, M. Matsumoto, M. Doi, FEBS Lett. 295 (1991) 67.

[50] M. Takahashi, K. Yoshikawa, V.V. Vasilevskaya, A.R. Khokhlov, J. Phys. Chem. 101 (1997) 9396.

[51] M.O. Khan, S.M. Mel'nikov, B. Jönsson, Macromolecules 32 (1999) 8836.

[52] M. Köping-Höggard, Y.S. Mel'nikova, K.M. Vårum, B. Lindman, P. Artursson, J. Gene Med. 5 (2003) 130.

[53] P.L. Felgner, T.R. Gadek, M. Holm, R. Roman, H.W. Chan, M Wenz, J.P. Northrop, G.M. Ringold, M. Danielsen, Proc. Natl. Acad. Sci. U.S.A. 84 (1987) 7413.

[54] D. Lasic, Liposomes in Gene Delivery, CRC Press, Boca Raton, FL, 1997.

[55] J. Rädler, I. Koltover, T. Salditt, C. Safinya, Science 275 (1997) 810.

[56] J.O. Rädler, I. Koltover, A. Jamieson, T. Salditt, C.R. Safinya, Langmuir 14 (1998) 4272.

[57] D.D. Lasic, H. Strey, M.C.A. Stuart, R. Podgornik, P.M.J. Frederik, J. Am. Chem. Soc. 119 (1997) 832. 
[58] R. Dias, B. Lindman, M. Miguel, J. Phys. Chem. B 106 (2002) 12600.

[59] K. Eskilsson, C. Leal, B. Lindman, M. Miguel, T. Nylander, Langmuir 17 (2001) 1666.

[60] M. Cardenás, A. Braem, T. Nylander, B. Lindman, Langmuir 19 (2003) 7712.

[61] Z.V. Leonenko, D.T. Cramb, NanoLetters 2 (2002) 305.

[62] S. Schounten, P. Stroeve, M.X. Lonogo, Langmuir 15 (1999) 8133.

[63] B. Maier, J.O. Rädler, Phys. Rev. Lett. 82 (1999) 1911.

[64] B. Maier, J.O. Rädler, Macromolecules 33 (2000) 7185.

[65] R.R. Netz, D. Andelman, Phys. Rep. 380 (2003) 1.

[66] T. Heimburg, B. Angerstein, D. Marsh, 76 (1999) 2575.

[67] W. Gottstein, S. Kreitmeier, M. Wittkip, D. Göritz, F. Gotsis, Polymers 7 (1997) 1607.

[68] M. Ellis, C.Y. Kong, C. Peterson, O. Sommelius, B. Söderberg, J. Chem. Phys. 112 (2000) 8723.

[69] C. Fleck, R.R. Netz, H.H. von Grünberg, Biophys. J. 82 (2002) 76.

[70] K. Holmberg, B. Jönsson, B. Kronberg, B. Lindman, Surfactants and Polymers in Aqueous Solution, 2nd ed., Wiley, New York, 2003.

[71] S.S. Sorlie, R. Pecora, Macromolecules 21 (1988) 1143.

[72] S.S. Sorlie, R. Pecora, Macromolecules 23 (1990) 487.

[73] R. Borsali, H. Nguyen, R. Pecora, Macromolecules 31 (1998) 1548.

[74] H. Liu, J. Gapinski, L. Skibinska, J. Chem. Phys. 113 (2000) 6001.

[75] M. Cárdenas, K. Schillén, T. Nylander, J. Jansson, B. Lindman, Phys. Chem. Chem. Phys. 6 (2004) 1603.

[76] S.M. Mel'nikov, V.G. Sergeyev, K. Yoshikawa, J. Am. Chem. Soc. 117 (1995) 2401.

[77] R.G. Winkler, M.O. Steinhauser, P. Reineker, Phys. Rev. E 66 (2002) 021802.

[78] Y. Hayashi, M. Ullner, P. Linse, J. Chem. Phys. 116 (2002) 6836.

[79] Y. Hayashi, M. Ullner, P. Linse, J. Phys. Chem. B 107 (2003) 8198.

[80] M. Ullner, B. Jönsson, C. Peterson, O. Sommelius, B. Söderberg, J. Chem. Phys. 107 (1997) 1279.
[81] M. Ullner, C.E. Woodward, Macromolecules 35 (2002) 1437.

[82] X. Shui, L. McFail-Isom, G.G. Hu, L.D. Williams, Biochemistry 37 (1998) 8341.

[83] S.S. Zakharova, S.U. Egelhaaf, L.B. Bhuiyan, C.W. Outhwaite, D. Bratko, J.R.C. van der Maarelet, J. Chem. Phys. 111 (1999) 10706

[84] H. Deng, V.A. Bloomfield, J.M. Benevides, G.J. Thomas Jr., Nucl. Acids Res. 28 (2000) 3379.

[85] A.P. Lyubartsev, L. Nordenskiöld, J. Chem. Phys. 101 (1997) 4335.

[86] N. Korolev, A.P. Lyubartsev, A. Laaksonen, L. Nordenskiöld, Biophys. J. 82 (2002) 2860

[87] A. Khan, E.F. Marques, in: I.D. Robb (Ed.), Specialists Surfactants, Blackie Academic, Professional, an imprint of Chapman \& Hall, London, 1997, p. 37.

[88] A. Khan, E. Marques, Curr. Opin. Colloid Interf. Sci. 4 (2000) 402.

[89] E. Marques, O. Regev, A. Khan, M. Miguel, B. Lindman, J. Phys. Chem. 102 (1998) 6746.

[90] M.Y. Yatcilla, K.L. Herrington, L.L. Brasher, E.W. Kaler, S. Chiruvolu, J.A. Zasadzinski, J. Phys. Chem. 100 (1996) 5874.

[91] L.L. Brasher, K.L. Herrington, E.W. Kaler, Langmuir 11 (1995) 4267.

[92] O. Regev, A. Khan, J. Colloid Interf. Sci. 182 (1996) 95.

[93] E. Kaler, K.L. Herrington, J.A.N. Zasadzinski, in: E.B. Sirota, D. Weitz, T. Witten, J. Israelachvili (Eds.), Complex Fluids, vol. 248, Material Research Society, Pittsburg, 1992, p. 3.

[94] S.M. Mel'nikov, R. Dias, Y. Mel'nikova, M. Marques, M. Miguel, B. Lindman, FEBS Lett. 453 (1999) 113.

[95] R.S. Dias, A.A.C.C. Pais, P. Linse, M.G. Miguel, B. Lindman, in preparation.

[96] R.S. Dias, Ph.D. Thesis, University of Coimbra, 2003.

[97] R.S. Dias, J. Innerlohinger, O. Glatter, M.G. Miguel, B. Lindman, in preparation.

[98] R.S. Dias, A.A.C.C. Pais, M.G. Miguel, B. Lindman, J. Chem. Phys. 119 (2003) 8150 